\title{
ESTABILIDADE ROBUSTA DE SISTEMAS LINEARES ATRAVÉS DE DESIGUALDADES MATRICIAIS LINEARES
}

\author{
Valter J. S. Leite* \\ valter@div.cefetmg.br \\ Paulo J. de Oliveira ${ }^{\ddagger}$ \\ jamesbkecampus 1 . uneb. br \\ Domingos C. W. Ramos ${ }^{\S}$ \\ domingos.ramos dembraer. com.br \\ Vinícius F. Montagner ${ }^{\dagger}$ \\ montagne@dt. fee. uni camp.br \\ Ricardo C. L. F. Oliveira ${ }^{\dagger}$ \\ ricfowddt. fee. unicamp.br \\ Pedro L. D. Peres ${ }^{\dagger}$ \\ peres@dt. fee.unicamp.br \\ ${ }^{*}$ UnED Divinópolis - CEFET-MG, R. Monte Santo, 319, 35502-036, Divinópolis - MG - Brasil \\ ${ }^{\dagger}$ Faculdade de Engenharia Elétrica e de Computação, Universidade Estadual de Campinas, CP 6101 \\ 13081-970, Campinas - SP - Brasil \\ $\ddagger$ DCET - UNEB, Av. Silveira Martins, 2555, 41195-001, Salvador - BA - Brasil \\ § EMBRAER - PC 392 - GME, Av. Brig. Faria Lima, 2170, 12227-901, São José dos Campos - SP - Brasil
}

\begin{abstract}
Sufficient conditions for the analysis of stability of linear systems with polytopic uncertainties are presented in this paper. The robust stability is guaranteed by the existence of a parameter dependent Lypaunov function obtained from the feasibility test of a set of linear matrix inequalities (LMIs) formulated at the vertices of the uncertainty polytope. Three conditions are presented, and the results are also compared with the analysis based on quadratic stability (same Lyapunov function for the entire set of uncertainties), for continuous as well as discrete-time systems. The first condition exploits the use of some extra variables (matrices) in the LMIs, and the second one uses a larger number of LMIs. These two conditions have recently appeared in the literature and are less conservative than quadratic stability. The third condition, proposed in this paper, combines the two ideas, yielding better results, and contains the previous conditions as particular cases. Several examples are presented to illustrate the
\end{abstract}

Artigo submetido em 13/03/03

1a. Revisão em 20/10/03

Aceito sob recomendação do Ed. Assoc. Prof. Liu Hsu numerical performance of the LMI conditions in terms of efficiency and computational complexity.

KEYWORDS: Robust stability, parameter dependent Lyapunov function, linear matrix inequality, polytopic uncertainty.

\section{RESUMO}

Condições suficientes para a análise de estabilidade de sistemas lineares com incertezas politópicas são apresentadas neste trabalho. A estabilidade robusta é garantida a partir da existência de uma função de Lyapunov dependente de parâmetros obtida do teste de factibilidade de um conjunto de desigualdades matriciais lineares (em inglês, LMIs — Linear Matrix Inequalities) formuladas nos vértices do politopo de incertezas. Três condições são apresentadas para sistemas contínuos no tempo, e três para sistemas a tempo discreto. Os resultados são também comparados com a análise baseada na estabilidade quadrática (mesma função de Lyapunov para todo o conjunto de incertezas), tanto para o caso contínuo quanto para o caso discreto. A primeira condição ex- 
plora o uso de variáveis (matrizes) adicionais em LMIs, e a segunda utiliza um número maior de LMIs. Essas duas condições foram recentemente publicadas e são menos conservadoras que a estabilidade quadrática. A terceira condição, proposta neste trabalho, mescla as duas idéias e apresenta resultados bem mais abrangentes, contendo as condições anteriores como casos particulares. Vários exemplos são apresentados, ilustrando o desempenho numérico das formulações LMI em termos de eficiência e de complexidade computacional.

PALAVRAS-CHAVE: Estabilidade robusta, função de Lyapunov dependente de parâmetro, desigualdade matricial linear, incerteza politópica.

\section{INTRODUÇÃO}

Uma das questões fundamentais na teoria de sistemas é a construção de funções de Lyapunov, tanto para a análise de estabilidade quanto para a síntese de controladores. Em particular, o estudo de sistemas lineares com parâmetros incertos avançou muito nas últimas duas décadas graças a técnicas de investigação de domínios de estabilidade e de controle robusto derivadas de funções de Lyapunov.

A chamada estabilidade quadrática (EQ), isto é, a existência de uma mesma função de Lyapunov, independente dos parâmetros incertos, assegurando a estabilidade robusta do sistema para o domínio de incertezas considerado, foi talvez o resultado mais importante da década de 80 (Barmish, 1985). A partir das condições de estabilidade quadrática, inúmeros resultados de análise, controle e filtragem robusta com critérios como as normas $\mathcal{H}_{2}$ e $\mathcal{H}_{\infty}$ puderam ser desenvolvidos (veja por exemplo (Boyd et al., 1994) e referências internas). Essas condições, quase sempre formuladas em termos de desigualdades matriciais lineares (em inglês, LMIs - Linear Matrix Inequalities), tornaram-se facilmente resolvíveis numericamente com o aparecimento de pacotes computacionais especializados (Gahinet et al., 1995).

Embora a estabilidade quadrática seja especialmente adequada à análise de sistemas incertos com parâmetros variantes no tempo, os resultados obtidos podem ser bastante conservadores em muitas situações. Recentemente, várias extensões têm aparecido na literatura para a análise e síntese de controladores para sistemas lineares incertos. Resultados menos conservadores têm sido obtidos a partir de funções de Lyapunov dependentes de parâmetros (Feron et al., 1996), (Gahinet et al., 1996), (Mori e Kokame, 2000), (Trofino, 1999), mas na maioria dos casos alguma restrição estrutural deve ser verificada, ou o procedimento numérico para o teste de estabilidade depende de parâmetros de escalonamentos ou de discretização do espaço paramétrico em uma malha suficientemente fina. Alguns trabalhos têm abor- dado o problema através de funções de Lyapunov quadráticas por partes (por exemplo (Rantzer e Johansson, 2000), (Xie et al., 1997)), mas a solução numérica, em geral, requer elevado esforço computacional.

Considerando apenas sistemas lineares incertos contínuos e invariantes no tempo, dentre os testes de estabilidade robusta baseados em funções de Lyapunov dependentes de parâmetros destacam-se, por estarem formulados em termos de LMIs e pela facilidade de resolução numérica, as abordagens apresentadas em (Geromel, de Oliveira e Hsu, 1998) e (Peaucelle et al., 2000). Em (Geromel, de Oliveira e Hsu, 1998), condições suficientes para a estabilidade estrutural de uma classe de sistemas foram propostas em termos de LMIs, sendo estabelecidas as conexões desses resultados com positividade real e passividade em sistemas lineares. Uma extensão desses resultados é apresentada em (Peaucelle et al., 2000), tratando diferentes regiões convexas dentro do plano complexo. A idéia principal contida nessas abordagens está no aumento da ordem das LMIs e na inclusão de novas variáveis, de maneira a obter, com esses graus de liberdade adicionais, resultados menos conservadores do que os conseguidos com a estabilidade quadrática. Nesses trabalhos, a estabilidade robusta é garantida por uma função de Lyapunov dependente de parâmetros, construída a partir de matrizes de Lyapunov que são soluções factíveis para um conjunto de LMIs descritas nos vértices do domínio de incertezas. Apesar de fornecer resultados melhores do que os obtidos com a estabilidade quadrática, essas condições (chamadas aqui de estabilidade robusta estendida - EE) ainda são conservadoras quando comparadas com o real domínio de estabilidade, provavelmente pelo fato de exigirem que uma ou mais variáveis satisfaçam conjuntamente todas as LMIs. Condições menos conservadoras (notadamente para casos de maiores dimensões) foram apresentadas em (Ramos e Peres, 2002), baseadas na construção apropriada de um número maior de LMIs descritas em função dos vértices do politopo de incertezas, denominadas neste trabalho de estabilidade robusta (ER).

Em relação a sistemas discretos no tempo, os testes de estabilidade evoluíram de maneira bastante similar. Dentre os testes baseados em funções de Lyapunov dependentes de parâmetros destacam-se as abordagens LMI apresentadas em (de Oliveira, Bernussou e Geromel, 1999), (de Oliveira, Geromel e Hsu, 1999) e (Peaucelle et al., 2000) (aumento da ordem das LMIs e inclusão de novas variáveis) e, mais recentemente, em (Ramos e Peres, 2001) (aumento do número de LMIs).

Este trabalho tem por base os artigos (Leite et al., 2002) e (de Oliveira et al., 2002), nos quais foram apresentadas as condições LMI de estabilidade robusta (ER) descritas acima, respectivamente, para os casos contínuo e discreto no tempo. 
Nesses artigos, uma exaustiva comparação numérica foi realizada, da qual conclui-se que a técnica que utiliza um número maior de LMIs (Ramos e Peres, 2001), (Ramos e Peres, 2002) produz, de maneira geral, avaliações menos conservadoras dos domínios de estabilidade quando comparada à que introduz novas variáveis (Peaucelle et al., 2000). É interessante comentar que ambas contêm a estabilidade quadrática como um caso particular (no sentido de que sempre fornecem uma solução factível quando o politopo de incertezas é quadraticamente estável), mas são no entanto independentes (ou seja, uma pode encontrar solução factível para um caso no qual a outra falha e vice-versa).

Neste trabalho, além da comparação numérica entre as condições LMI existentes na literatura, é apresentada uma nova condição suficiente, menos conservadora, para a estabilidade robusta de sistemas lineares com incertezas politópicas. A condição é formulada em termos de LMIs envolvendo apenas as matrizes associadas aos vértices do politopo (ou simplesmente matrizes vértices) de incertezas e, assim como as demais condições, pode ser testada por métodos de eficiência numérica comprovada e de complexidade polinomial (Gahinet et al., 1995). Essa condição, aqui denominada estabilidade combinada (EC), contém as anteriores como casos particulares.

Os casos contínuo e discreto no tempo são abordados. Uma exaustiva comparação numérica é feita entre os resultados obtidos com a estabilidade quadrática (EQ), a estabilidade robusta estendida (EE), a estabilidade robusta (ER) e com a condição aqui apresentada (EC) para um conjunto de politopos estáveis. Uma análise da complexidade numérica das LMIs envolvidas em cada tipo de teste também é apresentada. Além de mostrar que as condições EC produzem sempre os resultados menos conservadores, os exemplos ilustram a proximidade com as condições necessárias de estabilidade.

O artigo está organizado em duas partes, a primeira dedicada aos sistemas contínuos no tempo e a segunda aos sistemas a tempo discreto. O desenvolvimento de ambas é bastante semelhante. Primeiramente, expõe-se o problema e comentase sobre as condições clássicas de estabilidade. A seguir, as condições de estabilidade robusta são apresentadas, incluindo a condição mais geral aqui proposta. Uma análise da complexidade dos testes conclui as seções. Em seguida, exemplos numéricos são apresentados, e uma análise comparativa é feita. $\mathrm{O}$ artigo termina com a seção de conclusões.

\section{ESTABILIDADE DE SISTEMAS CONTÍ- NUOS NO TEMPO}

Considere um sistema linear contínuo no tempo descrito por

$$
\dot{x}(t)=A x(t)
$$

com $x \in \mathbb{R}^{n}$ e $A \in \mathbb{R}^{n \times n}$ uma matriz precisamente conhecida. Uma condição necessária e suficiente para a estabilidade assintótica de $A$, isto é, todos os autovalores de $A$ possuírem parte real estritamente negativa, é que exista $P=P^{\prime} \in \mathbb{R}^{n \times n}$ tal que as LMIs

$$
P>0 \quad ; \quad A^{\prime} P+P A<0
$$

sejam satisfeitas. Note que a desigualdade de Lyapunov acima poderia ser trocada, sem perda de generalidade, por $A^{\prime} P+P A \leq-\epsilon \mathbf{I}$ para qualquer $\epsilon>0$. Essa propriedade é muitas vezes chamada de homogeneidade.

Suponha agora que $A$ não é precisamente conhecida, mas pertence a um politopo de incertezas $\mathcal{A}$. Nesse caso, qualquer matriz $A$ dentro do domínio de incertezas pode ser escrita como a combinação convexa dos vértices $A_{j}, j=1, \ldots, N$, do politopo, ou seja, $A(\alpha) \in \mathcal{A}$ com

$$
\begin{aligned}
\mathcal{A}=\left\{A(\alpha) \in \mathbb{R}^{n \times n}: A(\alpha)=\sum_{j=1}^{N} \alpha_{j} A_{j}\right. \\
\left.\sum_{j=1}^{N} \alpha_{j}=1, \alpha_{j} \geq 0, j=1, \ldots, N\right\}
\end{aligned}
$$

Uma condição suficiente para a estabilidade do politopo $\mathcal{A}$ é dada pela existência de uma matriz de Lyapunov $P=P^{\prime} \in$ $\mathbb{R}^{n \times n}$ tal que as LMIs

$$
P>0 \quad ; \quad A(\alpha)^{\prime} P+P A(\alpha)<0
$$

sejam verificadas para todo $A(\alpha) \in \mathcal{A}$. Conhecida como estabilidade quadrática (EQ) na literatura (Barmish, 1985), essa condição pode ser facilmente verificada na prática graças à convexidade da desigualdade de Lyapunov, que faz com que a condição (4) seja equivalente à verificação da existência de $P=P^{\prime} \in \mathbb{R}^{n \times n}$ tal que

$$
P>0 \quad ; \quad A_{j}^{\prime} P+P A_{j}<0 ; j=1, \ldots, N
$$

Observe que (4) pode ser obtida de (5) multiplicando-se essa última por $\alpha_{j} \geq 0$ e somando em $j$, de $j=1$ até $j=N$.

Apesar de ser uma condição apenas suficiente para a estabilidade robusta do politopo $\mathcal{A}$, e que, portanto, pode produzir resultados conservadores, a estabilidade quadrática tem sido usada extensivamente para síntese de controladores e de filtros robustos nos mais diversos contextos, produzindo em muitos casos condições convexas para a determinação do controle ou do filtro. Veja por exemplo (Bernussou et al., 1989), (Geromel et al., 1991), (Khargonekar e Rotea, 1991), (Geromel, 1999), (Palhares e Peres, 1999), (de Souza e Trofino, 2000). 


\section{ESTABILIDADE ROBUSTA DE SISTE- MAS CONTÍNUOS NO TEMPO}

A análise da estabilidade do sistema incerto (1) com $A(\alpha) \in$ $\mathcal{A}$ pode ser efetuada através do uso de uma função de Lyapunov dependente do parâmetro incerto $\alpha$, ou seja, $A(\alpha)$ é assintoticamente estável se e somente se existir uma matriz de Lyapunov $P(\alpha)=P(\alpha)^{\prime}>0$ tal que

$$
A(\alpha)^{\prime} P(\alpha)+P(\alpha) A(\alpha)<0
$$

Note que o caso $P(\alpha)=P$ recai no clássico teste de estabilidade quadrática do sistema. Mesmo restringindo a busca de $P(\alpha)$ a funções de Lyapunov que se escrevem como uma combinação convexa de matrizes $P_{j}, j=1, \ldots, N$, condições menos conservadoras do que a estabilidade quadrática podem ser obtidas. Observe ainda que a existência de matrizes $P_{j}$ que verificam as desigualdades de Lyapunov nos vértices $A_{j}, j=1, \ldots, N$, do politopo $\mathcal{A}$ é apenas condição necessária para a estabilidade robusta. Note também que, embora a existência de $P(\alpha)=P(\alpha)^{\prime}>0$ que satisfaça (6) seja uma condição necessária e suficiente para a estabilidade de $A(\alpha)$, nada se pode afirmar a priori sobre a forma com que $P(\alpha)$ depende do parâmetro $\alpha$ (afim, polinomial, etc.).

Uma condição suficiente para a análise da estabilidade robusta de $A(\alpha)$ foi apresentada em (Geromel, de Oliveira e Hsu, 1998) e generalizada em (Peaucelle et al., 2000).

Lema 1 Se existirem $N$ matrizes simétricas definidas positivas $P_{j} \in \mathbb{R}^{n \times n}$ e matrizes $F \in \mathbb{R}^{n \times n}$ e $G \in \mathbb{R}^{n \times n}$ tais que

$$
\begin{aligned}
& {\left[\begin{array}{cc}
A_{j}^{\prime} F^{\prime}+F A_{j} & P_{j}-F+A_{j}^{\prime} G \\
P_{j}-F^{\prime}+G^{\prime} A_{j} & -\left(G+G^{\prime}\right)
\end{array}\right]<0} \\
& j=1, \ldots, N
\end{aligned}
$$

então $P(\alpha)=P(\alpha)^{\prime}>0$ dada por

$$
P(\alpha)=\sum_{j=1}^{N} \alpha_{j} P_{j}, \sum_{j=1}^{N} \alpha_{j}=1, \alpha_{j} \geq 0, j=1, \ldots, N
$$

é uma matriz de Lyapunov dependente do parâmetro $\alpha$ que assegura a estabilidade assintótica de qualquer $A(\alpha) \in \mathcal{A}$.

Prova: Veja (Peaucelle et al., 2000).

A idéia principal por trás do Lema 1 é que o aumento da dimensão do problema e a criação de novas variáveis (matrizes $F$ e $G$ ) propiciam um maior grau de liberdade para a verificação da condição (6) com $P(\alpha)$ dado por (8). Note que, com o aumento da dimensão da LMI, a matriz de Lyapunov $P$ não mais aparece envolvida em um produto com a matriz dinâmica $A$. É também interessante notar que, para $A$ fixo,

$$
\begin{aligned}
& A^{\prime} P+P A= \\
= & {\left[\begin{array}{c}
\mathbf{I} \\
A
\end{array}\right]^{\prime}\left[\begin{array}{cc}
A^{\prime} F^{\prime}+F A & P-F+A^{\prime} G \\
P-F^{\prime}+G^{\prime} A & -\left(G+G^{\prime}\right)
\end{array}\right]\left[\begin{array}{c}
\mathbf{I} \\
A
\end{array}\right] }
\end{aligned}
$$

para escolhas arbitrárias de $F$ e $G$, e que, portanto, (2) é verificada se e somente se a matriz interna do lado direito da equação (9) for definida negativa (note que $\left[\begin{array}{ll}\mathbf{I} & A^{\prime}\end{array}\right]$ tem posto completo). Até o presente, no entanto, não se conhecem possíveis extensões do Lema 1 para procedimentos de síntese menos conservadores do que os obtidos com estabilidade quadrática.

Embora a condição de estabilidade robusta estendida do Lema 1 forneça resultados melhores do que a estabilidade quadrática, a exigência de que as matrizes $F$ e $G$ devam satisfazer todo o conjunto de LMIs torna o resultado conservador, principalmente quando $N$ cresce. O lema a seguir apresenta uma nova condição suficiente para a análise da estabilidade robusta de $A(\alpha) \in \mathcal{A}$, publicada em (Ramos e Peres, 2002).

Lema 2 Se existirem $N$ matrizes simétricas definidas positivas $P_{j} \in \mathbb{R}^{n \times n}$ tais que

$$
\begin{gathered}
A_{j}^{\prime} P_{j}+P_{j} A_{j}<-\mathbf{I} ; j=1, \ldots, N \\
A_{j}^{\prime} P_{k}+P_{k} A_{j}+A_{k}^{\prime} P_{j}+P_{j} A_{k}<\frac{2}{N-1} \mathbf{I} \\
j=1, \ldots, N-1 ; k=j+1, \ldots, N
\end{gathered}
$$

então $P(\alpha)=P(\alpha)^{\prime}>0$ dada por (8) é uma função de Lyapunov dependente de parâmetros que assegura a estabilidade de qualquer $A(\alpha) \in \mathcal{A}$.

Prova: Primeiramente, note que $P(\alpha)$ dada por (8) com $P_{j}>0, j=1, \ldots, N$, é uma combinação convexa de matrizes definidas positivas e, portanto, também é definida positiva. Desenvolvendo a expressão (6), com $A(\alpha) \in \mathcal{A}$, obtémse

$$
\begin{aligned}
& A(\alpha)^{\prime} P(\alpha)+P(\alpha) A(\alpha)=\sum_{j=1}^{N} \alpha_{j}^{2}\left(A_{j}^{\prime} P_{j}+P_{j} A_{j}\right)+ \\
& +\sum_{j=1}^{N-1} \sum_{k=j+1}^{N} \alpha_{j} \alpha_{k}\left(A_{j}^{\prime} P_{k}+P_{k} A_{j}+A_{k}^{\prime} P_{j}+P_{j} A_{k}\right)
\end{aligned}
$$

Impondo as condições (10)-(11) (note que $\alpha_{j} \alpha_{k} \geq 0$ para todo $j$ e $k$ ), tem-se

$$
\begin{aligned}
& A(\alpha)^{\prime} P(\alpha)+P(\alpha) A(\alpha)< \\
& -\left(\sum_{j=1}^{N} \alpha_{j}^{2}-\sum_{j=1}^{N-1} \sum_{k=j+1}^{N} \alpha_{j} \alpha_{k} \frac{2}{N-1}\right) \mathbf{I} \leq 0
\end{aligned}
$$


pois a expressão dentro dos parênteses é sempre maior ou igual a zero. De fato,

$$
\begin{aligned}
& \sum_{j=1}^{N-1} \sum_{k=j+1}^{N}\left(\alpha_{j}-\alpha_{k}\right)^{2}= \\
& \quad=(N-1) \sum_{j=1}^{N} \alpha_{j}^{2}-2 \sum_{j=1}^{N-1} \sum_{k=j+1}^{N} \alpha_{j} \alpha_{k} \geq 0
\end{aligned}
$$

Vários pontos sobre o Lema 2 merecem ser comentados. A primeira observação diz respeito ao fato de que a condição $\sum_{j=1}^{N} \alpha_{j}=1$ não foi utilizada na demonstração. Embora se exija que os termos $\alpha_{j}$ sejam todos não negativos, não é necessário que a soma desses termos seja igual à unidade. $\mathrm{Na}$ verdade, basta apenas que os $\alpha_{j}$ não sejam todos nulos ou, equivalentemente, que $\sum_{j=1}^{N} \alpha_{j}=\rho, \rho>0$. Em outras palavras, se um politopo $\mathcal{A}$ é estável graças à verificação das condições do Lema 2, então qualquer combinação positiva dos vértices $A_{j}, j=1, \ldots, N$, produz também matrizes estáveis. Pode parecer surpreendente que um teste realizado nos vértices de um politopo conclua sobre a estabilidade de combinações lineares positivas desses mesmos vértices e, portanto, além da região delimitada pelo envelope convexo dos vértices. Mas se um politopo de matrizes, representando um sistema linear incerto contínuo no tempo, é estável, isto é, $A(\alpha)$ tem autovalores com parte real estritamente negativa para todo valor de $\alpha$, então qualquer combinação positiva dos vértices desse politopo também produz matrizes estáveis. Dessa forma, $\rho A(\alpha)$, para $\rho>0$, também terá autovalores com parte real negativa. As condições do Lema 2 reproduzem essa propriedade estrutural de politopos estáveis. Note que a condição de estabilidade quadrática, se verificada, também garante a estabilidade de qualquer combinação positiva $\sum_{j=1}^{N} \alpha_{j} A_{j}$, mas o mesmo não ocorre com a condição do Lema 1 .

Para sistemas precisamente conhecidos, isto é, para $N=1$, apenas a condição (10) precisa ser verificada para $P_{1}=$ $P_{1}^{\prime}>0$. Para sistemas incertos quadraticamente estáveis, existe uma matriz $P$ que verifica a condição (5) e, nesse caso, $P=P_{1}=P_{2}=\ldots=P_{N}$ é uma solução factível para (10)-(11). Note que se a equação (10) é satisfeita para uma mesma matriz $P$, então (11) é sempre verificada. Portanto as condições do Lema 2 englobam a condição de estabilidade quadrática como um caso particular.

A idéia central do Lema 2 está na exploração da homogeneidade da desigualdade de Lyapunov, impondo, sem perda de generalidade, que as desigualdades de Lyapunov devam ser menores que -I nos vértices do politopo (equação (10)). Esta condição, necessária para a estabilidade do politopo, permite que os termos cruzados assumam valores que podem até ser definidos positivos.
Utilizando de maneira combinada as idéias dos lemas 1 (variáveis extras) e 2 (LMIs extras), obtém-se uma condição menos conservadora, que contém as anteriores como casos particulares.

Lema 3 Se existirem matrizes simétricas definidas positivas $P_{j} \in \mathbb{R}^{n \times n}$, matrizes $F_{j} \in \mathbb{R}^{n \times n}$ e $G_{j} \in \mathbb{R}^{n \times n}$, $j=1, \ldots, N$ tais que

$$
\begin{aligned}
& {\left[\begin{array}{cc}
A_{j}^{\prime} F_{j}^{\prime}+F_{j} A_{j} & P_{j}-F_{j}+A_{j}^{\prime} G_{j} \\
P_{j}-F_{j}^{\prime}+G_{j}^{\prime} A_{j} & -\left(G_{j}+G_{j}^{\prime}\right)
\end{array}\right]<-\mathbf{I}} \\
& j=1, \ldots, N
\end{aligned}
$$

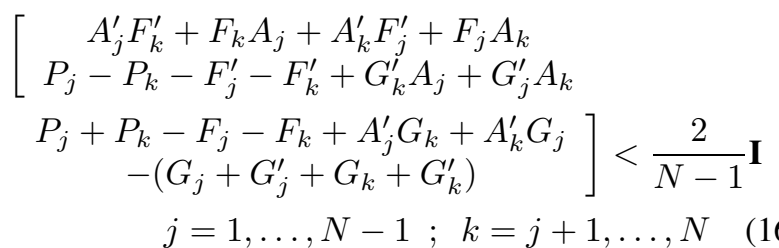

então com $G(\alpha)$ e $F(\alpha)$ dadas respectivamente por

$$
\begin{aligned}
G(\alpha)=\sum_{j=1}^{N} \alpha_{j} G_{j}, F(\alpha)=\sum_{j=1}^{N} \alpha_{j} F_{j} \\
\quad \sum_{j=1}^{N} \alpha_{j}=1, \alpha_{j} \geq 0, j=1, \ldots, N
\end{aligned}
$$

tem-se que $P(\alpha)=P(\alpha)^{\prime}>0$ dada por (8) é uma função de Lyapunov dependente do parâmetro $\alpha$ que assegura a estabilidade assintótica de qualquer $A(\alpha) \in \mathcal{A}$.

Prova: É similar à prova do Lema 2. Note que, com $P(\alpha)$, $F(\alpha)$ e $G(\alpha)$ dadas em (8) e (17), tem-se $P(\alpha)>0$ e

$$
\begin{gathered}
{\left[\begin{array}{c}
A(\alpha)^{\prime} F(\alpha)^{\prime}+F(\alpha) A(\alpha) \\
P(\alpha)-F(\alpha)^{\prime}+G(\alpha)^{\prime} A(\alpha) \\
P(\alpha)-F(\alpha)+A(\alpha)^{\prime} G(\alpha) \\
-\left(G(\alpha)+G(\alpha)^{\prime}\right)
\end{array}\right]=} \\
\quad=\sum_{j=1}^{N} \alpha_{j}^{2}\left[\begin{array}{cc}
A_{j}^{\prime} F_{j}^{\prime}+F_{j} A_{j} & P_{j}-F_{j}+A_{j}^{\prime} G_{j} \\
P_{j}-F_{j}^{\prime}+G_{j}^{\prime} A_{j} & -\left(G_{j}+G_{j}^{\prime}\right)
\end{array}\right]+ \\
+\sum_{j=1}^{N-1} \sum_{k=j+1}^{N} \alpha_{j} \alpha_{k}\left[\begin{array}{c}
A_{j}^{\prime} F_{k}^{\prime}+F_{k} A_{j}+A_{k}^{\prime} F_{j}^{\prime}+F_{j} A_{k} \\
P_{j}-P_{k}-F_{j}^{\prime}-F_{k}^{\prime}+G_{k}^{\prime} A_{j}+G_{j}^{\prime} A_{k} \\
P_{j}+P_{k}-F_{j}-F_{k}+A_{j}^{\prime} G_{k}+A_{k}^{\prime} G_{j} \\
-\left(G_{j}+G_{j}^{\prime}+G_{k}+G_{k}^{\prime}\right)
\end{array}\right]
\end{gathered}
$$

Impondo as condições (15)-(16), conclui-se que (veja a prova do Lema 2)

$$
\left[\begin{array}{c}
A(\alpha)^{\prime} F(\alpha)^{\prime}+F(\alpha) A(\alpha) \\
P(\alpha)-F(\alpha)^{\prime}+G(\alpha)^{\prime} A(\alpha) \\
P(\alpha)-F(\alpha)+A(\alpha)^{\prime} G(\alpha) \\
-\left(G(\alpha)+G(\alpha)^{\prime}\right)
\end{array}\right]<0
$$


Tabela 1: Número de variáveis escalares $K$ e de linhas $L$ das LMIs em função do número de estados $n$ e do número de vértices $N$ para os testes de estabilidade EQ (quadrática), EE (estendida), ER (robusta) e EC (combinada), caso contínuo. A complexidade é proporcional a $K^{3} L$ quando se utilizam métodos de pontos interiores.

\begin{tabular}{|c|c|c|}
\hline \hline Critério & $K$ (variáveis escalares) & $L$ (linhas) \\
\hline $\mathrm{EQ}$ & $\frac{n(n+1)}{2}$ & $(N+1) n$ \\
\hline $\mathrm{EE}$ & $\frac{N n(n+1)}{2}+2 n^{2}$ & $3 N n$ \\
\hline $\mathrm{ER}$ & $\frac{N n(n+1)}{2}$ & $\frac{N n(N+3)}{2}$ \\
\hline $\mathrm{EC}$ & $\frac{N n(5 n+1)}{2}$ & $N n(N+2)$ \\
\hline \hline
\end{tabular}

Multiplicando (19) à esquerda pela matriz $T(\alpha)=$ [ I $\left.A(\alpha){ }^{\prime}\right]$ e à direita por $T(\alpha)$, tem-se (6).

As LMIs do Lema 3 são condições suficientes para que (19) seja verificada, assumindo-se para isso que $P(\alpha), F(\alpha)$ e $G(\alpha)$ são expressas como a combinação convexa das matrizes vértices $P_{j}, F_{j}$ e $G_{j}, j=1, \ldots, N$. As condições do Lema 1 podem ser obtidas simplesmente fixando-se $F_{j}=F$, $G_{j}=G, j=1, \ldots, N$. Nesse caso, (16) torna-se redundante (sempre será satisfeita quando (15) for verificada). Também as condições do Lema 2 estão contidas nas LMIs do Lema 3, no bloco $(1,1)$, fazendo-se $F_{j}=F_{j}^{\prime}=P_{j}$ (nesse caso, as matrizes $G_{j}$ devem ser escolhidas adequadamente de maneira a garantir a factibilidade das LMIs).

As condições suficientes aqui apresentadas para a estabilidade robusta de um politopo de matrizes, ou seja, a estabilidade quadrática (EQ), a estabilidade robusta estendida do Lema 1 (EE), a estabilidade robusta do Lema 2 (ER) e a estabidade robusta combinada do Lema 3 (EC) expressam-se todas através de testes de factibilidade de um conjunto de LMIs. Hoje em dia há algoritmos eficientes baseados em métodos de pontos interiores de complexidade polinomial. A complexidade de problemas formulados em termos de LMIs está associada ao número $K$ de variáveis escalares e também com o número $L$ de linhas do sistema de equações. O número de operações em ponto flutuante ou o tempo necessário para se resolver um problema, utilizando-se métodos de pontos interiores do LMI Control Toolbox do Matlab (Gahinet et al., 1995), é proporcional a $K^{3} L$. Veja também (Boyd et al., 1994). A tabela 1 mostra os valores de $K$ e $L$ para cada um dos testes apresentados, sendo $n$ o número de estados do sistema e $N$ o número de vértices do politopo.

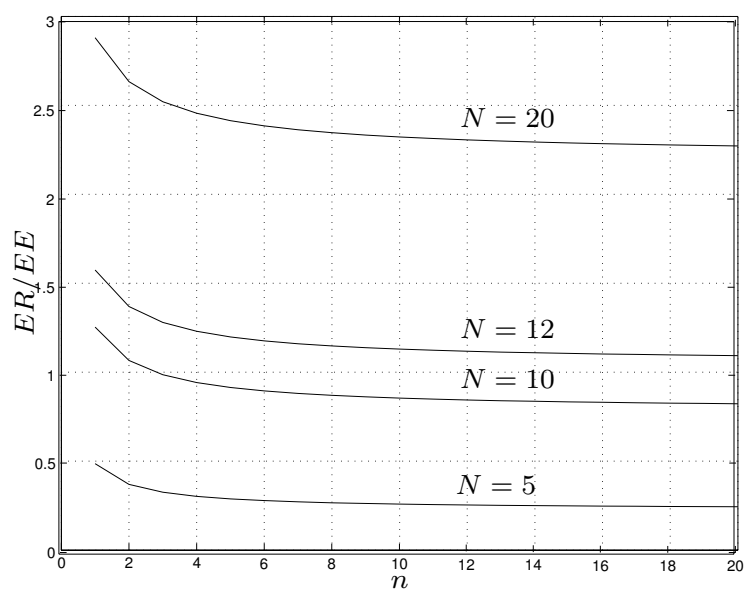

Figura 1: Complexidade numérica relativa ER/EE em função do número de estados $n$ (caso contínuo).

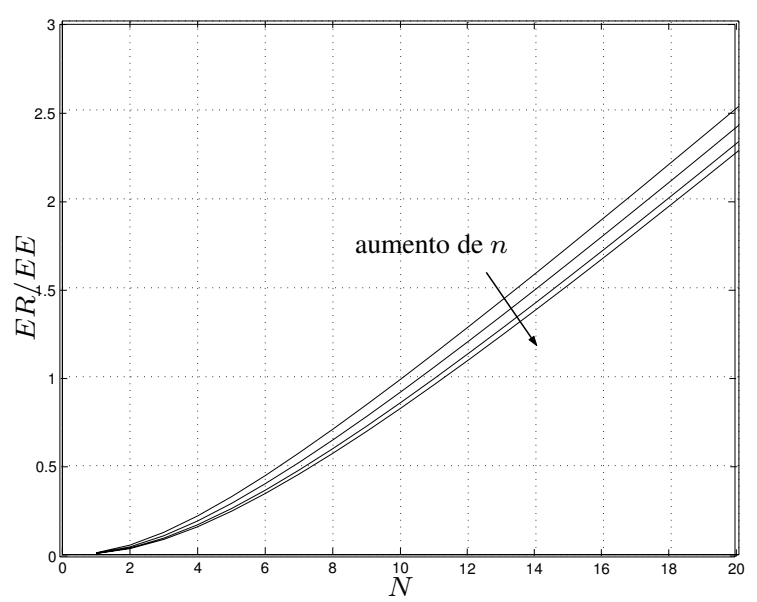

Figura 2: Complexidade numérica relativa ER/EE em função do número de vértices $N$ (caso contínuo).

A complexidade numérica do critério EQ é a menor, mas os resultados produzidos são bem mais conservadores. Uma comparação mais detalhada da complexidade dos métodos EE e ER, ambos menos conservadores que EQ e baseados em funções de Lyapunov dependentes de parâmetros, é mostrada nas figuras 1 e 2 . A figura 1 mostra a complexidade relativa ER/EE em função do número de estados $n$ para vários valores de $N$. Pode-se notar que a complexidade do critério ER torna-se maior que a do EE a partir de $N=12$. Na figura 2 é mostrada a complexidade relativa ER/EE em função do número de vértices. Pode-se observar que a influência de $N^{2}$ no critério ER torna-se o fator preponderante para valores grandes de $N(N>20)$, independente do número de estados $n$. Obviamente, o critério EC é o que apresenta a maior complexidade, pois possui mais variáveis escalares e mais linhas do 
que todos os demais.

\section{ESTABILIDADE DE SISTEMAS DISCRE- TOS NO TEMPO}

Considere o sistema linear discreto no tempo dado por

$$
x(k+1)=A x(k)
$$

com $x \in \mathbb{R}^{n}$ e $A \in \mathbb{R}^{n \times n}$ uma matriz precisamente conhecida. Uma condição necessária e suficiente para a estabilidade assintótica de $A$ (isto é, todos os autovalores de $A$ possuírem valores absolutos estritamente menores do que 1) é que exista $P=P^{\prime} \in \mathbb{R}^{n \times n}$ tal que as LMIs

$$
P>0 ; \quad A^{\prime} P A-P<0
$$

sejam satisfeitas. Note que a desigualdade de Lyapunov acima poderia ser trocada, sem perda de generalidade, por $A^{\prime} P A-P \leq-\epsilon \mathbf{I}$ para qualquer $\epsilon>0$ (homogeneidade da desigualdade de Lyapunov).

Suponha agora que $A$ não é precisamente conhecida, mas pertence a um politopo de incertezas $\mathcal{A}$. Nesse caso, qualquer matriz $A$ dentro do domínio de incertezas pode ser escrita como a combinação convexa dos vértices $A_{j}, j=1, \ldots, N$, do politopo, ou seja, $A(\alpha) \in \mathcal{A}$ dado por (3).

Uma condição suficiente para a estabilidade do politopo $\mathcal{A}$ é dada pela existência de uma matriz de Lyapunov $P=P^{\prime} \in$ $\mathbb{R}^{n \times n}$ tal que as LMIs

$$
P>0 \quad ; \quad A(\alpha)^{\prime} P A(\alpha)-P<0
$$

sejam verificadas para todo $A(\alpha) \in \mathcal{A}$. Conhecida como estabilidade quadrática (EQ) na literatura (Barmish, 1985), essa condição pode ser facilmente verificada na prática graças à convexidade da desigualdade de Lyapunov, que faz com que a condição (22) seja equivalente à verificação da existência de $P=P^{\prime} \in \mathbb{R}^{n \times n}$ tal que

$$
P>0 \quad ; \quad A_{j}^{\prime} P A_{j}-P<0 ; j=1, \ldots, N
$$

Tal fato pode ser melhor visualizado utilizando-se o complemento de Schur (Albert, 1969) aplicado à equação de Lyapunov discreta, que produz

$$
A_{j}^{\prime} P A_{j}-P<0 \Longleftrightarrow\left[\begin{array}{cc}
-P & P A_{j} \\
A_{j}^{\prime} P & -P
\end{array}\right]<0
$$

A estabilidade quadrática é condição apenas suficiente para a estabilidade robusta do politopo $\mathcal{A}$, podendo portanto produzir resultados conservadores. De maneira semelhante à que ocorre com sistemas contínuos, a estabilidade quadrática tem sido usada extensivamente para síntese de controladores e de filtros robustos nos mais diversos contextos, produzindo em muitos casos condições convexas para a determinação do controle ou do filtro. Veja por exemplo (Geromel, Bernussou, Garcia e de Oliveira, 1998), (Geromel et al., 1995), (Kaminer et al., 1993), (Palhares e Peres, 2000), (Palhares e Peres, 2001).

\section{ESTABILIDADE ROBUSTA DE SISTE- MAS DISCRETOS NO TEMPO}

A análise da estabilidade do sistema incerto (20) com $A(\alpha) \in \mathcal{A}$ pode ser efetuada através do uso de uma função de Lyapunov dependente do parâmetro incerto $\alpha$, ou seja, $A(\alpha)$ é assintoticamente estável se e somente se existir uma matriz de Lyapunov $P(\alpha)=P(\alpha)^{\prime}>0$ tal que

$$
A(\alpha)^{\prime} P(\alpha) A(\alpha)-P(\alpha)<0
$$

ou, equivalentemente,

$$
\left[\begin{array}{cc}
-P(\alpha) & P(\alpha) A(\alpha) \\
A(\alpha)^{\prime} P(\alpha) & -P(\alpha)
\end{array}\right]<0
$$

Mesmo restringindo a busca de $P(\alpha)$ a funções de Lyapunov que se escrevem como uma combinação convexa de matrizes, como em (8), condições menos conservadoras do que as da estabilidade quadrática (ou seja, $P(\alpha)=P$ em (25) ou em (26)) podem ser obtidas. Note que a existência de matrizes $P_{j}$ que verificam as desigualdades de Lyapunov nos vértices $A_{j}, j=1, \ldots, N$, do politopo $\mathcal{A}$ é apenas condição necessária para a estabilidade robusta. Assim como no caso contínuo, a existência de $P(\alpha)=P(\alpha)^{\prime}>0$ que satisfaça (25) é condição necessária e suficiente para a estabilidade de $A(\alpha)$, mas nada se pode afirmar sobre a estrutura de $P(\alpha)$.

Uma condição suficiente para a análise da estabilidade robusta de $A(\alpha)$, publicada em (de Oliveira, Bernussou e Geromel, 1999) e depois generalizada em (Peaucelle et al., 2000), é apresentada no Lema 4.

Lema 4 Se existirem $N$ matrizes simétricas definidas positivas $P_{j} \in \mathbb{R}^{n \times n}$ e matrizes $F \in \mathbb{R}^{n \times n}$ e $G \in \mathbb{R}^{n \times n}$ tais que

$$
\begin{aligned}
& {\left[\begin{array}{cc}
-P_{j}+A_{j}^{\prime} F^{\prime}+F A_{j} & -F+A_{j}^{\prime} G \\
-F^{\prime}+G^{\prime} A_{j} & P_{j}-\left(G+G^{\prime}\right)
\end{array}\right]<0} \\
& j=1, \ldots, N
\end{aligned}
$$

então $P(\alpha)=P(\alpha)^{\prime}>0$ dado por (8) é uma função de Lyapunov dependente do parâmetro $\alpha$ que assegura a estabilidade assintótica de qualquer $A(\alpha) \in \mathcal{A}$.

Prova: Veja (Peaucelle et al., 2000).

O Lema 4 explora o aumento da dimensão do problema e a criação de novas variáveis (matrizes $F$ e $G$ ) para a verificação da condição (25). De fato, é interessante notar que para 
$A$ fixo,

$$
\begin{aligned}
& A^{\prime} P A-P= \\
= & {\left[\begin{array}{c}
\mathbf{I} \\
A
\end{array}\right]^{\prime}\left[\begin{array}{cc}
-P+A^{\prime} F^{\prime}+F A & -F+A^{\prime} G \\
-F^{\prime}+G^{\prime} A & P-\left(G+G^{\prime}\right)
\end{array}\right]\left[\begin{array}{c}
\mathbf{I} \\
A
\end{array}\right] }
\end{aligned}
$$

para quaisquer $F$ e $G$. Fazendo $F=\mathbf{0}$, recupera-se o resultado apresentado em (de Oliveira, Bernussou e Geromel, 1999) para análise de estabilidade. Uma propriedade importante do Lema 4 é que a matriz $P$, utilizada para a análise de estabilidade, não aparece multiplicando a matriz $A$. Esse fato pode ser explorado para procedimentos de síntese, fazendo $F=\mathbf{0}$, substituindo $A^{\prime}$ por $A+B K$ e fazendo a mudança de variáveis $Z=K G$. Veja (de Oliveira, Bernussou e Geromel, 1999) para maiores detalhes.

A condição de estabilidade robusta estendida (EE) do Lema 4 fornece resultados menos conservadores do que a estabilidade quadrática (EQ), mas a exigência de que as matrizes $F$ e $G$ devam satisfazer todo o conjunto de LMIs deixa a condição ainda muito longe da necessidade, principalmente quando a dimensão do problema cresce.

Em (Ramos e Peres, 2001), uma nova condição suficiente para a análise da estabilidade robusta de $A(\alpha) \in \mathcal{A}$ foi apresentada, reproduzida no lema a seguir.

Lema 5 Se existirem $N$ matrizes simétricas definidas positivas $P_{j} \in \mathbb{R}^{n \times n}$ tais que

$$
\begin{gathered}
A_{j}^{\prime} P_{j} A_{j}-P_{j}<-\mathbf{I} \quad ; \quad j=1, \ldots, N \\
A_{j}^{\prime} P_{j} A_{k}+A_{k}^{\prime} P_{j} A_{j}+A_{j}^{\prime} P_{k} A_{j}-2 P_{j}-P_{k}<\frac{1}{(N-1)^{2}} \mathbf{I} \\
j=1, \ldots, N, k \neq j, k=1, \ldots, N \quad(30) \\
A_{j}^{\prime} P_{k} A_{\ell}+A_{\ell}^{\prime} P_{k} A_{j}+A_{k}^{\prime} P_{j} A_{\ell}+A_{\ell}^{\prime} P_{j} A_{k}+ \\
A_{j}^{\prime} P_{\ell} A_{k}+A_{k}^{\prime} P_{\ell} A_{j}-2\left(P_{j}+P_{k}+P_{\ell}\right)<\frac{6}{(N-1)^{2}} \mathbf{I} \\
j=1, \ldots, N-2, k=j+1, \ldots, N-1 \\
\ell=k+1, \ldots, N
\end{gathered}
$$

então $P(\alpha)=P(\alpha)^{\prime}>0$ dada por (8) é uma função de Lyapunov dependente de parâmetros que assegura a estabilidade de qualquer $A(\alpha) \in \mathcal{A}$.

Prova: Primeiramente, note que $P(\alpha)$ dada por (8) com $P_{j}>0, j=1, \ldots, N$, é uma combinação convexa de matrizes definidas positivas e, portanto, também é definida positiva. Desenvolvendo a expressão (25), com $A(\alpha) \in \mathcal{A}$, obtém-se

$$
\begin{gathered}
A(\alpha)^{\prime} P(\alpha) A(\alpha)-P(\alpha)= \\
=A(\alpha)^{\prime} P(\alpha) A(\alpha)-\left(\sum_{j=1}^{N} \alpha_{j}\right)^{2} P(\alpha)= \\
=\sum_{j=1}^{N} \alpha_{j}^{3}\left(A_{j}^{\prime} P_{j} A_{j}-P_{j}\right)+\sum_{j=1}^{N} \sum_{k \neq j ; k=1}^{N} \alpha_{j}^{2} \alpha_{k} \\
\left(A_{j}^{\prime} P_{j} A_{k}+A_{k}^{\prime} P_{j} A_{j}+A_{j}^{\prime} P_{k} A_{j}-2 P_{j}-P_{k}\right)+ \\
\sum_{j=1}^{N-2} \sum_{k=j+1}^{N-1} \sum_{\ell=k+1}^{N} \alpha_{j} \alpha_{k} \alpha_{\ell}\left(A_{j}^{\prime} P_{k} A_{\ell}+A_{\ell}^{\prime} P_{k} A_{j}+A_{k}^{\prime} P_{j} A_{\ell}\right. \\
\left.+A_{\ell}^{\prime} P_{j} A_{k}+A_{j}^{\prime} P_{\ell} A_{k}+A_{k}^{\prime} P_{\ell} A_{j}-2\left(P_{j}+P_{k}+P_{\ell}\right)\right)
\end{gathered}
$$

Impondo as condições (29)-(31) (note que $\alpha_{j} \geq 0$ para todo $j$ ), tem-se

$$
\begin{aligned}
A(\alpha)^{\prime} P(\alpha) A(\alpha)-P(\alpha)< & \\
- & \left(\sum_{j=1}^{N} \alpha_{j}^{3}-\frac{1}{(N-1)^{2}} \sum_{j=1}^{N} \sum_{k \neq j ; k=1}^{N} \alpha_{j}^{2} \alpha_{k}\right. \\
- & \left.\frac{6}{(N-1)^{2}} \sum_{j=1}^{N-2} \sum_{k=j+1}^{N-1} \sum_{\ell=k+1}^{N} \alpha_{j} \alpha_{k} \alpha_{\ell}\right) \mathbf{I} \leq 0
\end{aligned}
$$

pois a expressão dentro dos parênteses é sempre maior ou igual a zero. De fato, definindo $\Omega$ e $\Theta$ como

$$
\begin{gathered}
\Omega \triangleq \sum_{j=1}^{N} \sum_{k=1}^{N} \alpha_{j}\left(\alpha_{j}-\alpha_{k}\right)^{2}= \\
=(N-1) \sum_{j=1}^{N} \alpha_{j}^{3}-\sum_{j=1}^{N} \sum_{k \neq j ; k=1}^{N} \alpha_{j}^{2} \alpha_{k} \geq 0 \\
\Theta \triangleq \sum_{j=1}^{N} \sum_{k \neq j ; k=1}^{N-1} \sum_{\ell \neq j, k ; \ell=2}^{N} \alpha_{j}\left(\alpha_{k}-\alpha_{\ell}\right)^{2}= \\
=(N-2) \sum_{j=1}^{N} \sum_{k \neq j ; k=1}^{N} \alpha_{j}^{2} \alpha_{k} \\
-6 \sum_{j=1}^{N-2} \sum_{k=j+1}^{N-1} \sum_{\ell=k+1}^{N} \alpha_{j} \alpha_{k} \alpha_{\ell} \geq 0
\end{gathered}
$$

tem-se $(N-1) \Omega+\Theta \geq 0$. Veja (Ramos e Peres, 2001) para maiores detalhes.

Observe que, para sistemas precisamente conhecidos $(N=$ 1 ), apenas a condição (29) precisa ser verificada para $P_{1}=$ $P_{1}^{\prime}>0$. Note também que a condição (31) só precisa ser 
verificada para casos com $N \geq 3$. Para sistemas incertos quadraticamente estáveis, existe uma matriz $P$ que verifica a condição (23), e nesse caso $P=P_{1}=P_{2}=\cdots=P_{N}$ é uma solução factível para (29)-(31) (note que (29), nesse caso, implica que (30) e (31) são sempre satisfeitas), e portanto as condições do Lema 5 contêm a condição de estabilidade quadrática como um caso particular.

O Lema 5 explora a homogeneidade da desigualdade de Lyapunov, impondo sem perda de generalidade que as desigualdades de Lyapunov devam ser menores que -I nos vértices do politopo (condição (29), necessária para a estabilidade do politopo), permitindo assim que os termos cruzados assumam valores que podem até ser positivos. Os coeficientes foram então devidamente ajustados de maneira a garantir que o resultado final da soma descrita em (32) seja sempre uma matriz definida negativa, independentemente dos valores de $\alpha$.

Nas condições do Lema 5 utiliza-se a equação discreta de Lyapunov em sua forma original (25). É interessante notar que outras condições suficientes para a estabilidade robusta (isto é, para a verificação da equação (25)) poderiam ser obtidas a partir da forma de Schur dada em (26). De fato,

$$
\begin{aligned}
& {\left[\begin{array}{cc}
-P(\alpha) & P(\alpha) A(\alpha) \\
A(\alpha)^{\prime} P(\alpha) & -P(\alpha)
\end{array}\right]=} \\
& =\sum_{j=1}^{N} \alpha_{j}^{2}\left[\begin{array}{cc}
-P_{j} & P_{j} A_{j} \\
A_{j}^{\prime} P_{j} & -P_{j}
\end{array}\right]+ \\
& +\sum_{j=1}^{N-1} \sum_{k=j+1}^{N} \alpha_{j} \alpha_{k}\left[\begin{array}{cc}
-P_{j}-P_{k} & P_{j} A_{k}+P_{k} A_{j} \\
A_{j}^{\prime} P_{k}+A_{k}^{\prime} P_{j} & -P_{j}-P_{k}
\end{array}\right]
\end{aligned}
$$

e, de maneira similar ao que foi feito nos lemas 2 e 3, a expressão (36) será definida negativa se forem impostas as restrições

$$
\begin{gathered}
{\left[\begin{array}{cc}
-P_{j} & P_{j} A_{j} \\
A_{j}^{\prime} P_{j} & -P_{j}
\end{array}\right]<-\mathbf{I} ; j=1, \ldots, N} \\
{\left[\begin{array}{cc}
-P_{j}-P_{k} & P_{j} A_{k}+P_{k} A_{j} \\
A_{j}^{\prime} P_{k}+A_{k}^{\prime} P_{j} & -P_{j}-P_{k}
\end{array}\right]<\frac{2}{N-1} \mathbf{I}} \\
j=1, \ldots, N-1 ; k=j+1, \ldots, N
\end{gathered}
$$

A forma de Schur equivalente às condições do Lema 5 poderia ser obtida multiplicando-se ambos os lados de (36) por $\sum_{j=1}^{N} \alpha_{j}$, em uma manipulação similar à que será apresentada no próximo lema. Essas condições, assim como (37)(38), estão no entanto contidas na formulação mais geral do Lema 6.

Assim como no caso contínuo, o uso combinado das variáveis extras do Lema 4 e das LMIs adicionais do Lema 5 permitem a formulação de uma nova condição suficiente, que fornece resultados menos conservadores e que contém as condições dos dois lemas anteriores.

Lema 6 Se existirem matrizes simétricas definidas positivas $P_{j} \in \mathbb{R}^{n \times n}$, matrizes $F_{j} \in \mathbb{R}^{n \times n}$ e $G_{j} \in \mathbb{R}^{n \times n}$, $j=1, \ldots, N$ tais que

$$
\begin{aligned}
& {\left[\begin{array}{cc}
-P_{j}+A_{j}^{\prime} F_{j}^{\prime}+F_{j} A_{j} & -F_{j}+A_{j}^{\prime} G_{j} \\
-F_{j}^{\prime}+G_{j}^{\prime} A_{j} & P_{j}-\left(G_{j}+G_{j}^{\prime}\right)
\end{array}\right]<-\mathbf{I}} \\
& j=1, \ldots, N
\end{aligned}
$$

$$
\begin{aligned}
& {\left[F_{j} A_{j}+A_{j}^{\prime} F_{j}^{\prime}+F_{j} A_{k}+A_{k}^{\prime} F_{j}^{\prime}\right.} \\
& +F_{k} A_{j}+A_{j}^{\prime} F_{k}^{\prime}-2 P_{j}-P_{k} \\
& \left(G_{j}+G_{k}\right) A_{j}+G_{j} A_{k}-2 F_{j}^{\prime}-F_{k}^{\prime} \\
& \left.\begin{array}{l}
A_{j}^{\prime}\left(G_{j}^{\prime}+G_{k}^{\prime}\right)+A_{k}^{\prime} G_{j}^{\prime}-2 F_{j}-F_{k} \\
2 P_{j}+P_{k}-2 G_{j}-2 G_{j}^{\prime}-G_{k}-G_{k}^{\prime}
\end{array}\right]<\frac{1}{(N-1)^{2}} \mathbf{I} \\
& j=1, \ldots, N ; k \neq j ; k=1, \ldots, N
\end{aligned}
$$

$$
\left[\begin{array}{c}
F_{j} A_{k}+A_{k}^{\prime} F_{j}^{\prime}+F_{j} A_{\ell}+A_{\ell}^{\prime} F_{j}^{\prime} \\
+F_{k} A_{j}+A_{j}^{\prime} F_{k}^{\prime}+F_{k} A_{\ell}+A_{\ell}^{\prime} F_{k}^{\prime} \\
+F_{\ell} A_{j}+A_{j}^{\prime} F_{\ell}^{\prime}+F_{\ell} A_{k} \\
+A_{k}^{\prime} F_{\ell}^{\prime}-2\left(P_{j}+P_{k}+P_{\ell}\right) \\
G_{k} A_{j}+G_{\ell} A_{j}+G_{j} A_{k}+G_{\ell} A_{k} \\
+G_{j} A_{\ell}+G_{k} A_{\ell}-2\left(F_{j}+F_{k}+F_{\ell}\right)^{\prime}
\end{array}\right.
$$

$$
\begin{gathered}
A_{j}^{\prime} G_{k}^{\prime}+A_{j}^{\prime} G_{\ell}^{\prime}+A_{k}^{\prime} G_{j}^{\prime}+A_{k}^{\prime} G_{\ell}^{\prime} \\
+A_{\ell}^{\prime} G_{j}^{\prime}+A_{\ell}^{\prime} G_{k}^{\prime}-2\left(F_{j}+F_{k}+F_{\ell}\right) \\
2\left(P_{j}+P_{k}+P_{\ell}\right) \\
-2\left(G_{j}+G_{j}^{\prime}+G_{k}+G_{k}^{\prime}+G_{\ell}+G_{\ell}^{\prime}\right) \\
j=1, \ldots, N-2 \\
<\frac{6}{(N-1)^{2}} \mathbf{I} ; \quad k=j+1, \ldots, N-1 \\
\ell=k+1, \ldots, N
\end{gathered}
$$

então, com $G(\alpha)$ e $F(\alpha)$ dadas por (17), tem-se que $P(\alpha)=$ $P(\alpha)^{\prime}>0$ dada por (8) é uma função de Lyapunov dependente do parâmetro $\alpha$ que assegura a estabilidade assintótica de qualquer $A(\alpha) \in \mathcal{A}$.

Prova: É bastante similar às demonstrações anteriores. $P(\alpha)$ dada por (8) é definida positiva e, a partir das restrições (39)-(41) e das definições de $\Omega$ e $\Theta$ em (34)-(35) (veja a equação (33)), conclui-se que

$$
\left[\begin{array}{c}
-P(\alpha)+A(\alpha)^{\prime} F(\alpha)^{\prime}+F(\alpha) A(\alpha) \\
-F(\alpha)^{\prime}+G(\alpha)^{\prime} A(\alpha) \\
-F(\alpha)+A(\alpha)^{\prime} G(\alpha) \\
P(\alpha)-\left(G(\alpha)+G(\alpha)^{\prime}\right)
\end{array}\right]<0
$$


A condição de estabilidade robusta (25) é recuperada multiplicando-se (42) à esquerda por $T(\alpha)=\left[\begin{array}{ll}\mathbf{I} & A(\alpha)^{\prime}\end{array}\right]$ e à direita por $T(\alpha)^{\prime}$.

As LMIs do Lema 6 são condições suficientes para que (42) seja verificada, assumindo-se para isso que $P(\alpha), F(\alpha)$ e $G(\alpha)$ são expressas como a combinação convexa das matrizes vértices $P_{j}, F_{j}$ e $G_{j}, j=1, \ldots, N$. As condições do Lema 4 podem ser obtidas simplesmente fixando-se $F_{j}=F$, $G_{j}=G, j=1, \ldots, N$. Nesse caso, (40) e (41) tornam-se redundantes (sempre serão satisfeitas quando (39) for verificada). Também as condições do Lema 5, em uma formulação Schur equivalente, estão contidas nas LMIs do Lema 6 , fazendo-se $F_{j}=\mathbf{0}$ e $G_{j}=G_{j}^{\prime}=P_{j}$.

Assim como no caso contínuo, as condições de estabilidade robusta de um politopo de matrizes aqui apresentadas baseiam-se em testes de factibilidade de um conjunto de LMIs. A tabela 2 mostra os valores de $K$ e $L$ (a complexidade é proporcional a $K^{3} L$, quando são usados métodos de pontos interiores como os do LMI Control Toolbox do Matlab (Gahinet et al., 1995)) para cada um dos testes relativos a sistemas discretos no tempo, sendo $n$ o número de estados do sistema e $N$ o número de vértices do politopo.

Tabela 2: Número de variáveis escalares $K$ e de linhas $L$ das LMIs em função do número de estados $n$ e do número de vértices $N$ para os testes de estabilidade EQ (quadrática), EE (estendida), ER (robusta) e EC (combinada), caso discreto. A complexidade é proporcional a $K^{3} L$ quando se utilizam métodos de pontos interiores.

\begin{tabular}{|c|c|c|}
\hline \hline Critério & $K$ (variáveis) & $L$ (linhas) \\
\hline $\mathrm{EQ}$ & $\frac{n(n+1)}{2}$ & $(N+1) n$ \\
\hline $\mathrm{EE}$ & $\frac{N n(n+1)}{2}+2 n^{2}$ & $3 N n$ \\
\hline $\mathrm{ER}$ & $\frac{N n(n+1)}{2}$ & $\frac{N n\left(N^{2}+3 N+8\right)}{6}$ \\
\hline $\mathrm{EC}$ & $\frac{N n(5 n+1)}{2}$ & $\frac{N n\left(N^{2}+3 N+5\right)}{3}$ \\
\hline \hline
\end{tabular}

A complexidade numérica do critério EQ é a menor, mas os resultados produzidos são bem mais conservadores. A complexidade do critério ER é menor que a do EE para $N \leq 5$ e maior para $N>6$, independentemente de $n$. Para $N=8$ e $n \geq 3$ a complexidade associada a ER é cerca de duas vezes maior que a do critério EE. Como ocorreu no caso contínuo, o teste EC (que engloba os demais resultados) é o de maior complexidade, apresentando um número de linhas nas LMIs que cresce com $N^{3}$ e que rapidamente torna-se o fator

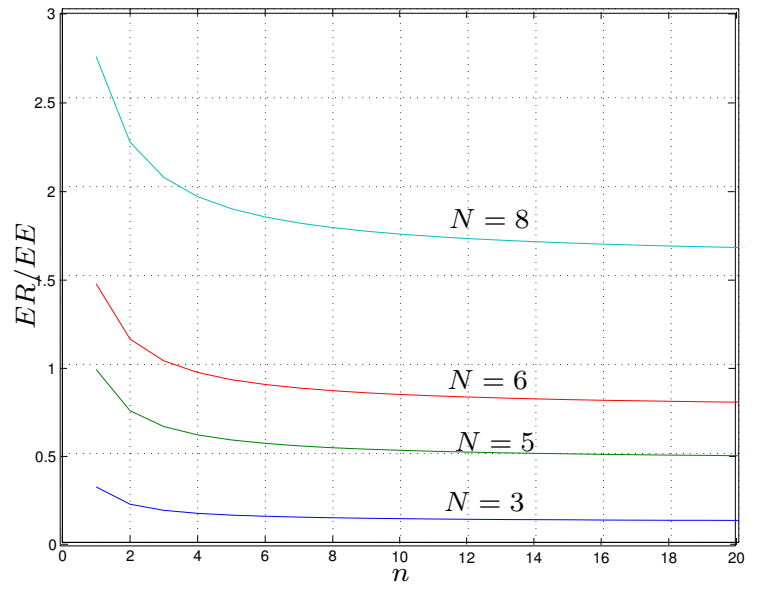

Figura 3: Complexidade numérica relativa ER/EE em função do número de estados $n$ (caso discreto).

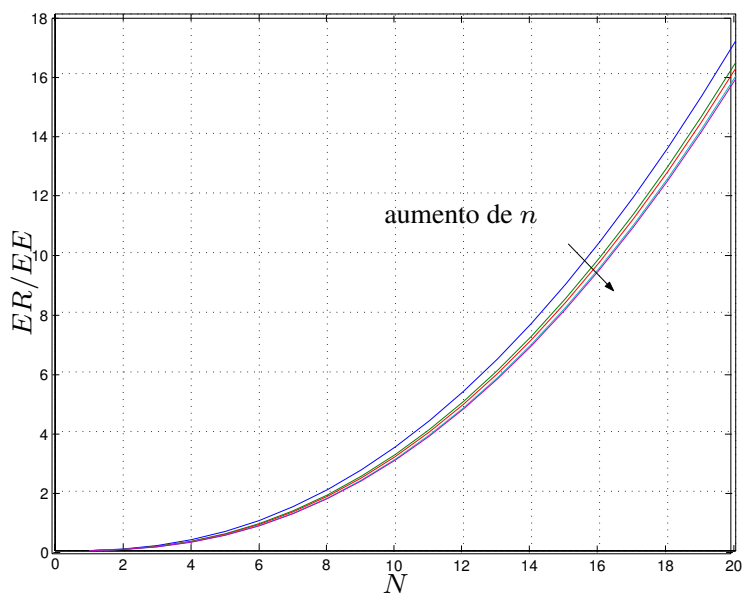

Figura 4: Complexidade numérica relativa ER/EE em função do número de vértices $N$ (caso discreto).

dominante. As figuras 3 e 4 mostram o comportamento da complexidade relativa $\mathrm{ER} / \mathrm{EE}$.

Como comentários finais, antes da apresentação dos resultados numéricos, duas observações sobre melhoria das condições apresentadas (em termos de torná-las menos conservadoras), ao custo do aumento da complexidade dos problemas, são pertinentes e aplicam-se tanto ao caso contínuo quanto ao discreto no tempo. Uma primeira estratégia diz respeito ao uso de matrizes cujos elementos também serão variáveis do problema de otimização, no lugar das matrizes constantes iguais a I do lado direito das LMIs, por exemplo em (10)-(11), caso contínuo, ou em (29)-(31), caso discreto, incluindo restrições adicionais para que (13), caso contínuo, e (33), caso discreto, sejam respeitadas. A segunda possibilidade vem da multiplicação, por exemplo, dos dois lados 
de (12) no caso contínuo ou de (32) no discreto pelo fator $\left(\sum_{j=1}^{N} \alpha_{j}\right)^{d}=1$, para $d$ inteiro, gerando assim novas condições que podem ser exploradas de maneira similar à exposta neste trabalho. Essas e outras melhorias nas condições apresentadas são atualmente objeto de estudo por parte dos autores.

\section{EXEMPLOS}

\subsection{Sistemas Contínuos}

Primeiramente, foi feita uma comparação exaustiva entre os critérios de estabilidade quadrática EQ, estabilidade estendida EE, estabilidade robusta ER e estabilidade combinada EC (lembrando que este método, além de sempre fornecer resultados melhores, contém os anteriores como casos particulares). Para isso, foram gerados de maneira aleatória 1000 politopos estáveis para cada par $(n, N)$ com $2 \leq n \leq 5$ e $2 \leq N \leq 5$. O número 1000 foi escolhido por ter sido verificado que os percentuais de sucesso de cada método não sofrem alterações significativas quando números maiores de politopos são investigados.

A geração dos politopos estáveis foi realizada adotando-se os seguintes passos: (i) geração de $N$ matrizes de dimensões $n \times n$ cujos elementos são valores reais uniformemente distribuídos no intervalo $[-1,1]$; (ii) para cada vértice, determinação da maior parte real dos autovalores, $\sigma$, e subtração de $\left(\sigma+1 \times 10^{-4}\right) \mathbf{I}_{n}$, garantindo pelo menos um autovalor de cada vértice com parte real em $-1 \times 10^{-4}$; (iii) determinação da maior das partes reais de todos os autovalores do politopo gerado, $\sigma_{\max }$, através de uma malha fina; (iv) subtração de $\left(\sigma_{\max }+1 \times 10^{-4}\right) \mathbf{I}_{n}$ de cada uma das matrizes vértices do politopo.

No total, foram gerados 16000 politopos estáveis, e o resultado dos testes de estabilidade está apresentado na tabela 3. O critério quadrático mostra resultados mais conservadores, e essa tendência acentua-se com o aumento no número de vértices independentemente do número de estados. O teste de estabilidade robusta proposto no Lema 3 (EC) apresenta os melhores resultados, com avaliações positivas sempre que EE ou ER fornecem solução factível e, em inúmeras situações (principalmente quando a complexidade aumenta) é a única condição que identifica a estabilidade dos politopos.

Comparando-se as condições EE e ER, pode-se dizer que, em termos gerais, o critério ER é o menos conservador, apresentando no entanto resultados similares e até um pouco piores que os do critério EE para valores pequenos de $N$ e $n$ (veja também (Leite et al., 2002) para uma comparação apenas entre EQ, EE e ER). Note que as condições EE e ER contêm EQ mas não estão contidas uma na outra (são condições suficientes independentes). A figura 5 mostra a evolução dos

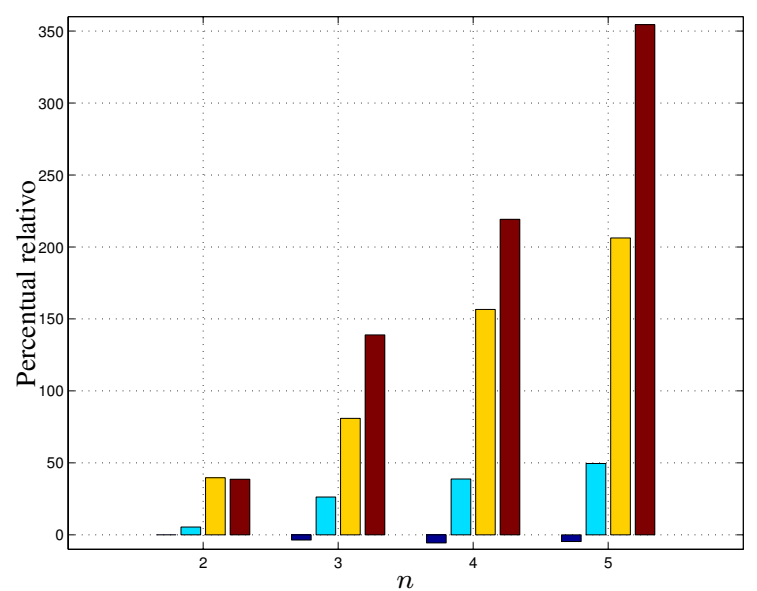

Figura 5: Percentual relativo de acertos ER/EE em função do aumento do número de estados $n$ para grupos de exemplos com número de vértices $N$ variando de 2 a 5 , da esquerda para a direita. Caso contínuo.

percentuais da diferença entre o número de acertos de ER e o número de acertos de EE em relação ao método que obteve menor índice absoluto. Esses percentuais são apresentados em função do aumento da complexidade dos casos, $n$ crescendo de 2 a 5 nas abscissas e, para cada valor de $n$, o número de vértices $N$ variando de 2 a 5 , da esquerda para a direita.

Uma visualização dos resultados da tabela 3 é dada na figura 6, na qual são mostrados quatro gráficos na forma de barras comparando o número de politopos estáveis identificados por cada método. Os eixos das abscissas referem-se ao número de estados do politopo, cada qual com um grupo de quatro barras verticais que representam o número de politopos identificados como estáveis. A barra mais à esquerda corresponde a $N=2$ e a mais à direita a $N=5$.

Finalmente, para se ter uma idéia melhor da flagrante superioridade do método do Lema 3 (EC) em relação aos demais, foi feita a comparação relativa entre EC e a melhor dentre as condições EE e ER. O resultado está mostrado na Figura 7 (valores positivos significam melhor desempenho de ER em relação a EE).

Um exemplo simples, também gerado aleatoriamente, com $n=2$ e $N=3$, ilustra muito bem como um politopo estável é resolvido pelos critérios ER e EC e não verifica nem a condição de estabilidade quadrática EQ nem a condição de Lema 1 (estabilidade EE). Os vértices do politopo são dados pelas matrizes

$$
\begin{aligned}
& A_{1}=\left[\begin{array}{cc}
-3.100 & 4.700 \\
-8.200 & -13.300
\end{array}\right] \\
& A_{2}=\left[\begin{array}{cc}
-1.300 & -2.100 \\
9.100 & 1.200
\end{array}\right]
\end{aligned}
$$


Tabela 3: Número de politopos estáveis identificados pelos testes EQ (estabilidade quadrática), EE (estabilidade estendida), ER (estabilidade robusta) e EC (estabilidade combinada) quando aplicados a 1000 politopos estáveis para $2 \leq n \leq 5$, $2 \leq N \leq 5$. Sistemas contínuos.

\begin{tabular}{|c|c|c|c|c|c|c|c|c|c|c|c|}
\hline$n$ & $\bar{N}$ & EQ & $\overline{\mathrm{EE}}$ & ER & $\overline{\mathrm{EC}}$ & $n$ & $\overline{N N}$ & EQ & 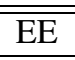 & $\overline{E \text { ER }}$ & EC \\
\hline \multirow{4}{*}{2} & 2 & 642 & 1000 & 1000 & 1000 & & 2 & 346 & 959 & 925 & 959 \\
\hline & 3 & 378 & 948 & 1000 & 1000 & 3 & 3 & 133 & 681 & 860 & 927 \\
\hline & 4 & 361 & 711 & 993 & 998 & & 4 & 106 & 461 & 834 & 918 \\
\hline & 5 & 361 & 719 & 997 & 1000 & & 5 & 93 & 360 & 860 & 920 \\
\hline \multirow{4}{*}{4} & 2 & 229 & 969 & 915 & 969 & & 2 & 226 & 973 & 928 & 973 \\
\hline & 3 & 65 & 611 & 848 & 931 & 5 & 3 & 28 & 573 & 857 & 941 \\
\hline & 4 & 32 & 318 & 816 & 907 & & 4 & 22 & 257 & 787 & 908 \\
\hline & 5 & 42 & 255 & 814 & 903 & & 5 & 14 & 169 & 768 & 904 \\
\hline
\end{tabular}
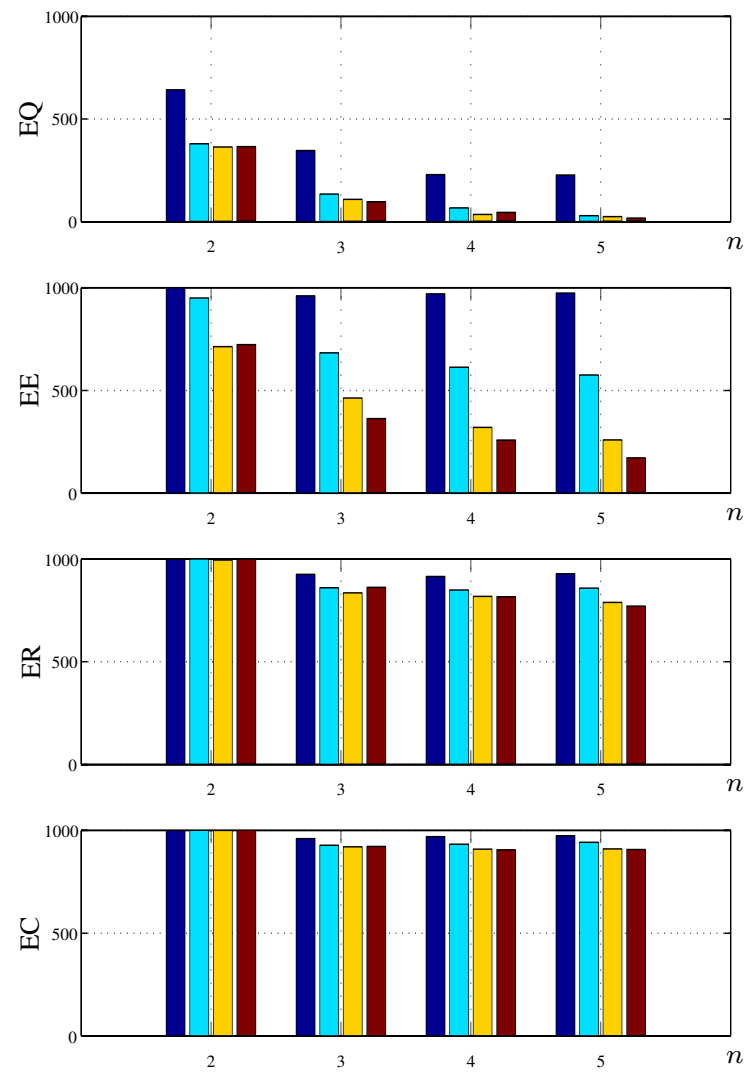

Figura 6: Número de politopos estáveis identificados pelos critérios EQ, EE, ER e EC, para $2 \leq n \leq 5$ (abscissas) e $2 \leq N \leq 5$ (barras verticais, $N=2$ à esquerda). Sistemas contínuos.

$$
A_{3}=\left[\begin{array}{ll}
0.300 & -6.000 \\
9.500 & -1.500
\end{array}\right]
$$

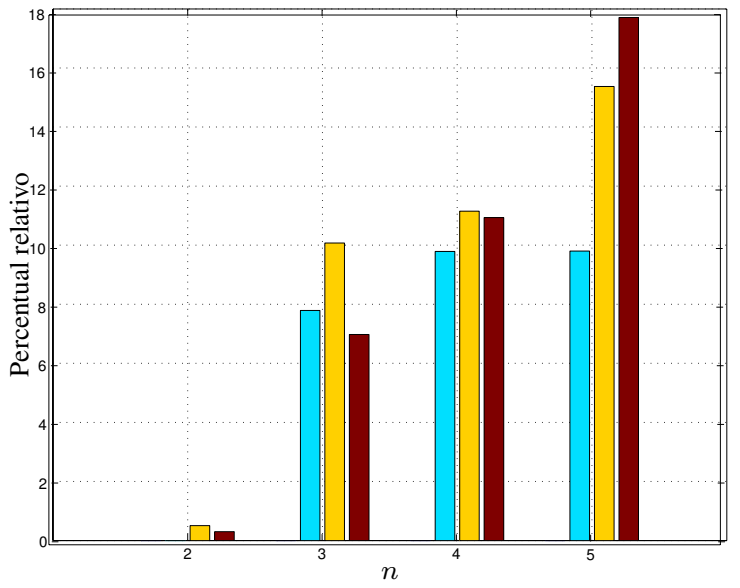

Figura 7: Percentual relativo de acertos EC/(melhor entre EE e ER) em função do aumento do número de estados $n$ para grupos de exemplos com número de vértices $N$ variando de 3 a 5 , da esquerda para a direita, caso contínuo. Não houve ganho para $N=2, n=2,3,4,5$ nem tampouco para $N=3$, $n=2$.

e a região de estabilidade é ilustrada na figura 8 .

Também usando geração aleatória de politopos estáveis pode-se encontrar exemplos relativamente simples que são identificados como estáveis apenas pelo método do Lema 3 (EC), como o sistema incerto dado pelos vértices

$$
\begin{aligned}
& A_{1}=\left[\begin{array}{ccc}
-0.502 & 0.560 & -0.179 \\
0.708 & -0.593 & 0.122 \\
0.589 & 0.023 & -0.233
\end{array}\right] \\
& A_{2}=\left[\begin{array}{ccc}
-0.410 & 0.159 & -0.689 \\
-0.839 & -0.779 & -0.200 \\
0.460 & 0.666 & -0.158
\end{array}\right]
\end{aligned}
$$




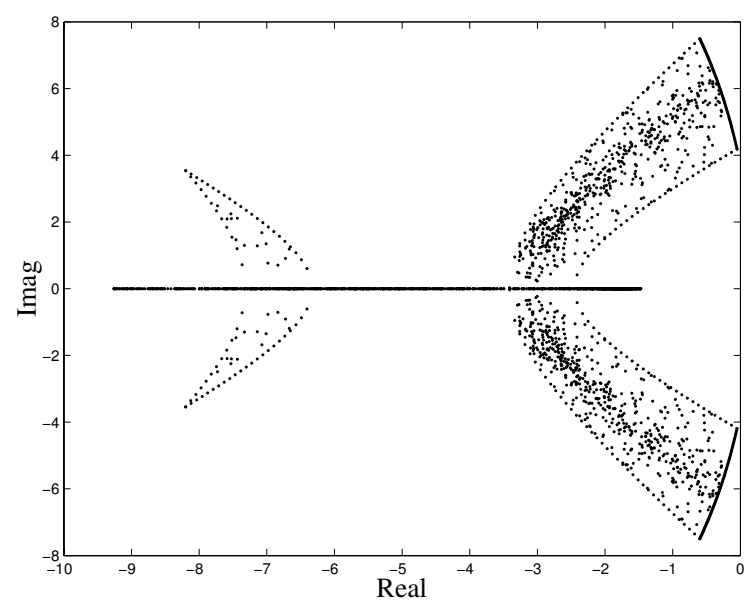

Figura 8: Nuvem de raízes do sistema contínuo dado por (43), identificado como estável apenas pelas condições ER e EC.

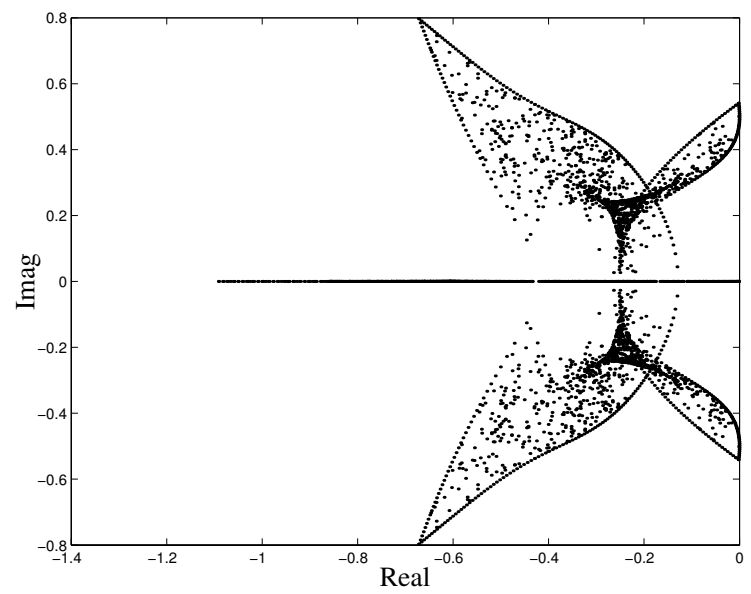

Figura 9: Nuvem de raízes do sistema contínuo dado por (44), identificado como estável apenas pela condição EC.

$$
A_{3}=\left[\begin{array}{ccc}
-0.808 & -0.845 & 0.207 \\
0.672 & 0.641 & -0.423 \\
-0.912 & -0.090 & -0.575
\end{array}\right]
$$

O lugar das raízes está ilustrado na Figura 9.

Um teste de estabilidade com $n=2$ e $N=2$ dura menos do que $70 \mathrm{~ms}$, independentemente do método. Para $n=5$ e $N=5$ o tempo médio foi de $80 \mathrm{~ms}$ (EQ), $0.31 \mathrm{~s}$ (EE), $0.31 s(\mathrm{ER})$ e $133 s$ (EC). Os testes foram realizados em um computador AMD K7 ATHLON 1.4 GHz, com 256 Mbytes de memória RAM, usando Matlab e o pacote LMI Control Toolbox (Gahinet et al., 1995). O teste EC é sempre o mais lento, mas esse aumento de complexidade é o preço a ser pago para obter resultados menos conservadores.

\subsection{Sistemas Discretos}

Assim como no caso contínuo, foi feita uma comparação exaustiva entre os métodos apresentados para identificação de sistemas incertos discretos no tempo. Para isso, foram também gerados 1000 politopos estáveis para cada par $(n, N) \operatorname{com} 2 \leq n \leq 5$ e $2 \leq N \leq 5$.

A geração dos politopos estáveis foi realizada adotando-se a seguinte estratégia: ( $i$ ) geração de $N$ matrizes de dimensões $n \times n$ cujos elementos são valores reais uniformemente distribuídos no intervalo $[-1,1]$; (ii) determinação, para cada vértice, do maior autovalor em módulo $\sigma$; (iii) divisão de cada matriz vértice pelo respectivo $\sigma / 0.95$, de maneira a garantir que, inicialmente, cada vértice possua pelo menos um autovalor com módulo igual a 0.95 ; (iv) determinação do maior dos valores absolutos de todos os autovalores do politopo gerado, $\sigma_{\max }$, através de uma malha fina; (iii) divisão de cada uma das matrizes vértices do politopo por $\left(\sigma_{\max } / 0.99\right)$, garantindo que a nuvem de autovalores possui pelo menos um elemento com módulo igual a 0.99 .

No total, foram gerados 16000 politopos estáveis, e o resultado dos testes de estabilidade está apresentado na tabela 4.

O critério quadrático mostra resultados bem mais conservadores, praticamente só identicando politopos estáveis para $N=2$ e $N=3$ vértices, e mesmo assim com um percentual bastante baixo. Note que os politopos estáveis gerados sempre estão no limite da estabilidade, com autovalores muito próximos do círculo unitário. Uma visualização dos resultados da tabela 4 é dada na figura 10 , na qual são mostrados quatro gráficos na forma de barras comparando o número de politopos estáveis identificados por cada método. Os eixos das abscissas referem-se ao número de estados do politopo, cada qual com um grupo de quatro barras verticais que representam o número de politopos identificados como estáveis. A barra mais à esquerda corresponde a $N=2$ e a mais à direita a $N=5$.

$\mathrm{Na}$ comparação entre as condições independentes EE e ER, nota-se uma ligeira vantagem para EE (problemas de baixa complexidade) e um desempenho melhor de ER quando $n$ e $N$ aumentam. Os percentuais relativos entre o número de acertos de ER e o número de acertos de EE em relação ao que obteve menor índice absoluto são mostrados na figura 11. Esses percentuais são apresentados em função do aumento da complexidade dos casos, $n$ crescendo de 2 a 5 nas abscissas e, para cada valor de $n$, o número de vértices $N$ variando de 2 a 5 , da esquerda para a direita. Valores positivos indicam que ER identificou mais politopos estáveis do que EE. É importante salientar, entretanto, que EE e ER são condições apenas suficientes, e que não estão contidas uma na outra, mas ambas estão contidas em EC. Veja (de Oliveira et al., 2002) para mais detalhes da comparação entre EQ, EE e ER. 
Tabela 4: Número de politopos estáveis identificados pelos testes EQ (estabilidade quadrática), EE (estabilidade estendida), ER (estabilidade robusta) e EC (estabilidade combinada) quando aplicados a 1000 politopos estáveis para cada par $2 \leq n \leq 5$, $2 \leq N \leq 5$. Sistemas discretos.

\begin{tabular}{|c|c|c|c|c|c|c|c|c|c|c|c|}
\hline $\bar{n}$ & 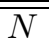 & 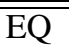 & 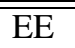 & 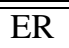 & 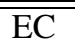 & $\overline{\bar{n} n}$ & 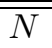 & 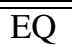 & 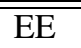 & 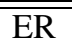 & $\overline{\overline{\mathrm{EC}}}$ \\
\hline \multirow{4}{*}{2} & 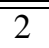 & 326 & "996 & 8881 & "996 & \multirow{4}{*}{3} & $\overline{2}$ & 186 & 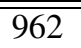 & 8699 & $\overline{9662}$ \\
\hline & 3 & 55 & 890 & 761 & 989 & & 3 & 5 & 751 & 714 & 920 \\
\hline & 4 & 10 & 789 & 646 & 984 & & 4 & 1 & 524 & 578 & 855 \\
\hline & 5 & 1 & 692 & 545 & 979 & & 5 & 0 & 427 & 508 & 862 \\
\hline \multirow{4}{*}{4} & 2 & 110 & 956 & 873 & 956 & \multirow{4}{*}{5} & 2 & 79 & 963 & 880 & 963 \\
\hline & 3 & 4 & 716 & 705 & 895 & & 3 & 0 & 748 & 747 & 918 \\
\hline & 4 & 1 & 460 & 605 & 857 & & 4 & 0 & 400 & 584 & 847 \\
\hline & 5 & 0 & 288 & 506 & 838 & & 5 & 0 & 220 & 468 & 813 \\
\hline
\end{tabular}
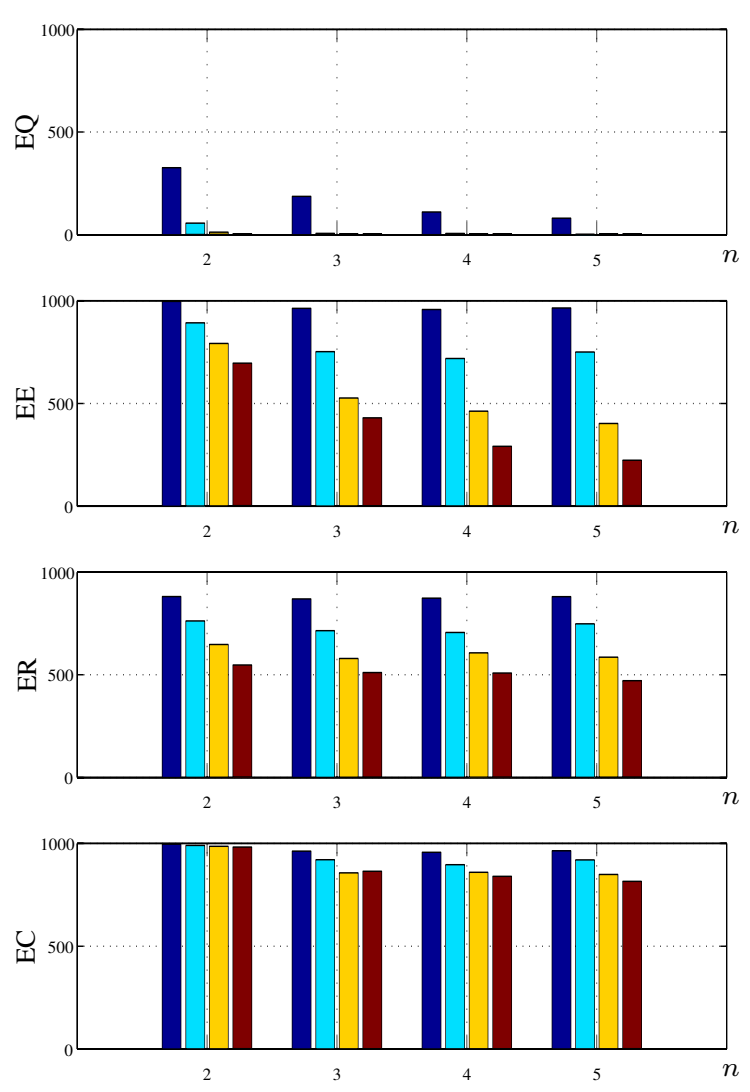

Figura 10: Número de politopos estáveis identificados pelos critérios EQ, EE, ER e EC, para $2 \leq n \leq 5$ (abscissas) e $2 \leq N \leq 5$ (barras verticais, $N=2$ à esquerda). Sistemas discretos.

A comparação relativa do método EC com a melhor dentre as duas condições (EE e ER) mostrada na Figura 12 deixa

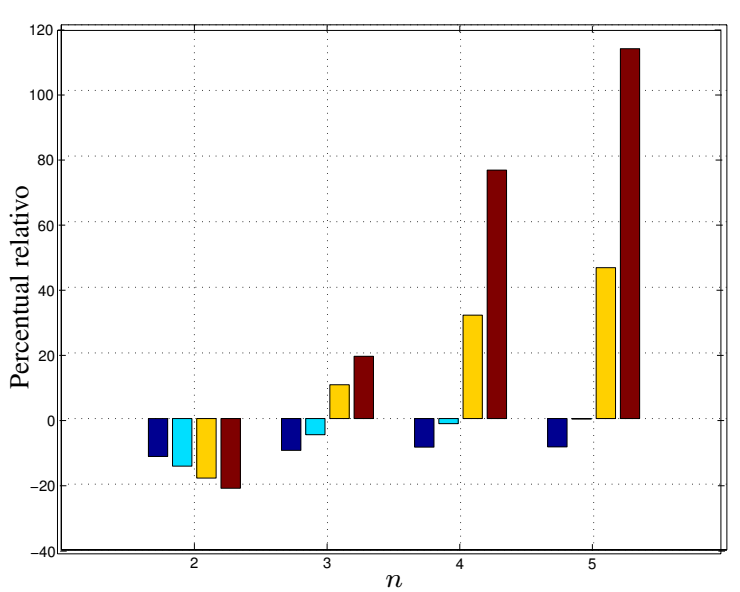

Figura 11: Percentual relativo de acertos ER/EE em função do aumento do número de estados $n$ para grupos de exemplos com número de vértices $N$ variando de 2 a 5 , da esquerda para a direita. Caso discreto.

patente a superioridade do resultado do Lema 6.

Lembrando que EE e ER são condições independentes, e que portanto uma pode identificar um politopo como estável e a outra não ou vice-versa, foi gerado aleatoriamente um exemplo simples para o qual EE falha, mas ER e EC são verificadas. Os vértices do politopo são dados por

$$
\begin{aligned}
& A_{1}=\left[\begin{array}{cc}
-0.800 & -0.200 \\
1.200 & 1.100
\end{array}\right] \\
& A_{2}=\left[\begin{array}{cc}
0.900 & -0.600 \\
0.000 & 0.300
\end{array}\right] \\
& A_{3}=\left[\begin{array}{cc}
-0.500 & -0.200 \\
0.900 & -1.300
\end{array}\right]
\end{aligned}
$$

cujo lugar das raízes está representado na Figura 13. 


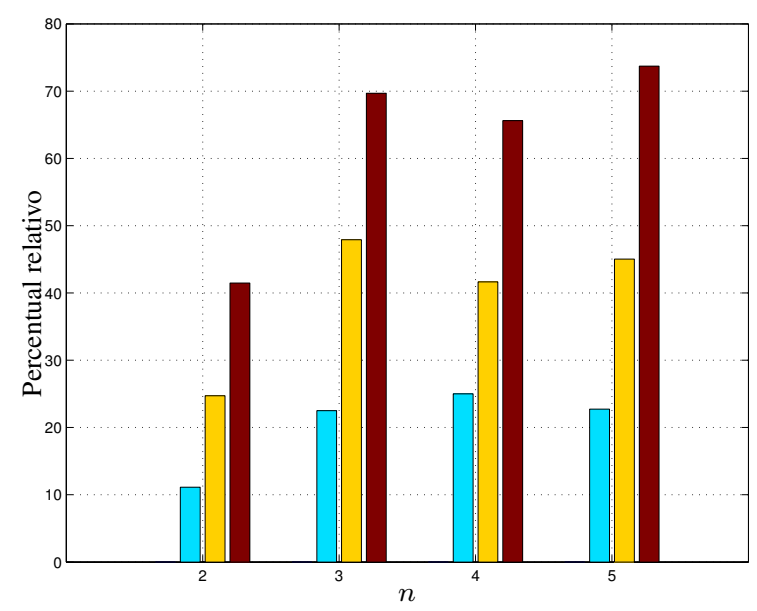

Figura 12: Percentual relativo de acertos EC/(melhor entre EE e ER) em função do aumento do número de estados $n$ para grupos de exemplos com número de vértices $N$ variando de 2 a 5 , da esquerda para a direita, caso discreto. Não houve ganho para $N=2, n=2,3,4,5$.

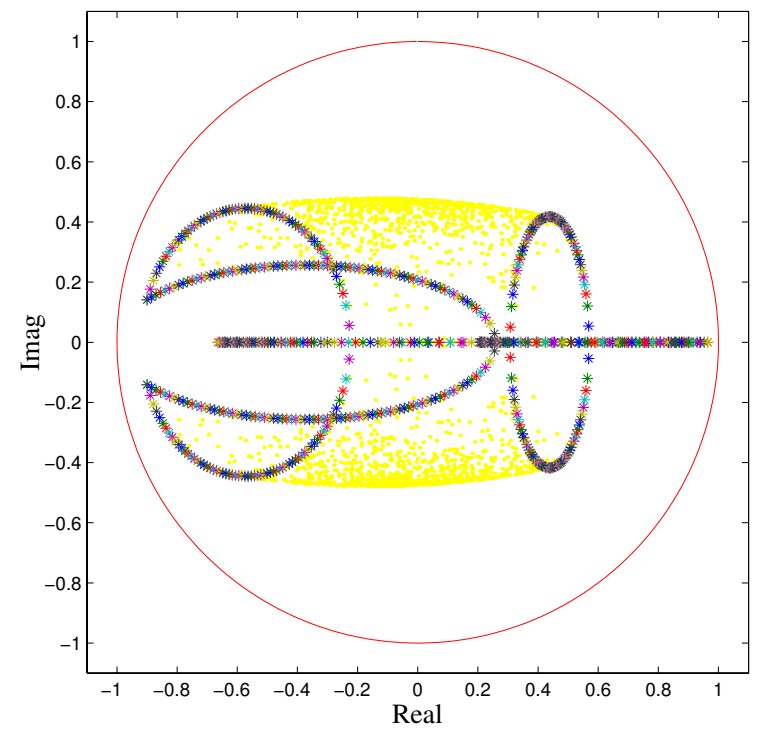

Figura 13: Nuvem de raízes associada ao sistema discreto dado por (45), identificado como estável apenas pelas condições ER e EC.

O politopo descrito pelos vértices

$$
\begin{aligned}
& A_{1}=\left[\begin{array}{ccc}
0.143 & 0.656 & -0.590 \\
-0.813 & 0.216 & -0.015 \\
0.649 & -0.681 & -0.418
\end{array}\right] \\
& A_{2}=\left[\begin{array}{ccc}
1.303 & 0.636 & -0.147 \\
-0.617 & -0.041 & 0.978 \\
-0.244 & -1.120 & -0.537
\end{array}\right]
\end{aligned}
$$

$$
A_{3}=\left[\begin{array}{ccc}
-0.495 & 0.438 & 0.895 \\
-0.188 & -1.113 & -0.295 \\
0.559 & 0.275 & 0.041
\end{array}\right]
$$

no entanto só foi identificado como estável pelo Lema 6 (EC). O lugar das raízes está na Figura 14.

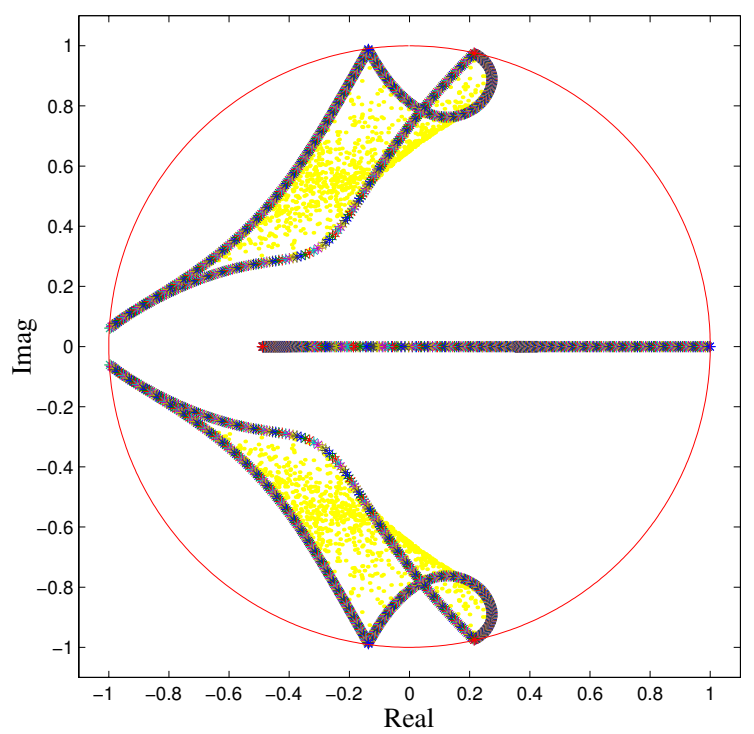

Figura 14: Nuvem de raízes associada ao sistema discreto dado por (46), identificado como estável apenas pela condição EC.

O caso discreto exige, em geral, maior tempo de processamento do que o caso contínuo. Um teste de estabilidade com $n=2$ e $N=2$ exige menos do que $70 \mathrm{~ms}$ independentemente do método utilizado. Para $n=5$ e $N=5$ o tempo médio é de $65 \mathrm{~ms}$ (EQ), $0.48 \mathrm{~s}$ (EE), $1.89 \mathrm{~s}$ (ER) e $75 \mathrm{~s}$ (EC). Foi utilizado o computador AMD K7 ATHLON $1.4 \mathrm{GHz}$, com 256 Mbytes de memória RAM, e Matlab com o pacote LMI Control Toolbox (Gahinet et al., 1995). Como no caso contínuo, o teste que exige maior tempo de processamento é o que envolve a aplicação das condições EC, as quais, em contrapartida, identificam um número bem superior de politopos estáveis quando comparadas aos testes das demais condições (EQ, EE e ER).

\section{CONCLUSÃO}

Este trabalho apresentou e comparou condições para testes de estabilidade robusta de um politopo de matrizes em termos de LMIs, tanto para sistemas a tempo contínuo como para sistemas discretos no tempo. Além de recapitular condições clássicas baseadas na estabilidade quadrática (mesma função de Lyapunov para todo o domínio de incertezas) e condições recentes da literatura baseadas em funções de Lyapunov dependentes de parâmetros, foram apresentadas novas 
condições para o caso contínuo e para o caso discreto, também formuladas em termos de LMIs, que não só contêm as anteriores como casos particulares mas também são capazes de identicar um número substancialmente maior de politopos estáveis, como mostrado na análise numérica e nos exemplos apresentados. Essas condições menos conservadoras, embora computacionalmente mais complexas, também podem ser testadas através de algoritmos especializados em LMIs (por exemplo, utilizando métodos de pontos interiores) em tempo polinomial.

A partir dos resultados apresentados, diversas extensões e generalizações podem ser aventadas, como por exemplo o cômputo de custos garantidos $\mathcal{H}_{2}$ e $\mathcal{H}_{\infty}$, a síntese de controladores dependentes de parâmetros, o tratamento de incertezas variantes no tempo, temas no momento sob investigação por parte dos autores.

\section{AGRADECIMENTOS}

Os autores agradecem o apoio das agências CAPES, CNPq, FAPESP e FAPEMIG (TEC 1233/98).

\section{REFERÊNCIAS}

Albert, A. (1969). Conditions for positive and nonnegative definiteness in terms of pseudoinverses, SIAM Journal on Applied Mathematics 17(2): 434-440.

Barmish, B. R. (1985). Necessary and sufficient conditions for quadratic stabilizability of an uncertain system, Journal of Optimization Theory and Applications 46(4): 399-408.

Bernussou, J., Peres, P. L. D. e Geromel, J. C. (1989). A linear programming oriented procedure for quadratic stabilization of uncertain systems, Systems \& Control Letters 13(1): 65-72.

Boyd, S., El Ghaoui, L., Feron, E. e Balakrishnan, V. (1994). Linear Matrix Inequalities in System and Control Theory, SIAM Studies in Applied Mathematics, Philadelphia, PA.

de Oliveira, M. C., Bernussou, J. e Geromel, J. C. (1999). A new discrete-time robust stability condition, Systems \& Control Letters 37(4): 261-265.

de Oliveira, M. C., Geromel, J. C. e Hsu, L. (1999). LMI characterization of structural and robust stability: the discrete-time case, Linear Algebra and Its Applications 296(1-3): 27-38.

de Oliveira, P. J., Oliveira, R. C. L. F., Leite, V. J. S., Montagner, V. F. e Peres, P. L. D. (2002). Estabilidade robusta de sistemas discretos no tempo através de desigualdades matriciais lineares, XIV Congresso Brasileiro de Automática, Natal, RN, pp. 2884-2889.

de Souza, C. E. e Trofino, A. (2000). A linear matrix inequality approach to the design of robust $\mathcal{H}_{2}$ filters, in $\mathrm{L}$. El Ghaoui e S. I. Niculescu (eds), Advances in Linear Matrix Inequality Methods in Control, Advances in Design and Control, SIAM, Philadelphia, PA, pp. 175-185.

Feron, E., Apkarian, P. e Gahinet, P. (1996). Analysis and synthesis of robust control systems via parameterdependent Lyapunov functions, IEEE Transactions on Automatic Control 41(7): 1041-1046.

Gahinet, P., Apkarian, P. e Chilali, M. (1996). Affine parameter-dependent Lyapunov functions and real parametric uncertainty, IEEE Transactions on Automatic Control 41(3): 436-442.

Gahinet, P., Nemirovski, A., Laub, A. J. e Chilali, M. (1995). LMI Control Toolbox for use with Matlab, User's Guide, The Math Works Inc., Natick, MA.

Geromel, J. C. (1999). Optimal linear filtering under parameter uncertainty, IEEE Transactions on Signal Processing 47(1): 168-175.

Geromel, J. C., Bernussou, J., Garcia, G. e de Oliveira, M. C. (1998). $\mathcal{H}_{2}$ and $\mathcal{H}_{\infty}$ robust filtering for discrete-time linear systems, Proceedings of the 37th IEEE Conference on Decision and Control, Vol. 1, Tampa, FL, pp. 632637.

Geromel, J. C., de Oliveira, M. C. e Hsu, L. (1998). LMI characterization of structural and robust stability, Linear Algebra and Its Applications 285(1-3): 69-80.

Geromel, J. C., Peres, P. L. D. e Bernussou, J. (1991). On a convex parameter space method for linear control design of uncertain systems, SIAM Journal on Control and Optimization 29(2): 381-402.

Geromel, J. C., Peres, P. L. D. e Souza, S. R. (1995). A convex approach to the mixed $\mathcal{H}_{2} / \mathcal{H}_{\infty}$ control problem for discrete-time uncertain systems, SIAM Journal on Control and Optimization 33(6): 1816-1833.

Kaminer, I., Khargonekar, P. P. e Rotea, M. A. (1993). Mixed $\mathcal{H}_{2} / \mathcal{H}_{\infty}$ control for discrete-time systems via convex optimization, Automatica 29(1): 57-70.

Khargonekar, P. P. e Rotea, M. A. (1991). Mixed $\mathcal{H}_{2} / \mathcal{H}_{\infty}$ control: a convex optimization approach, IEEE Transactions on Automatic Control 36(7): 824-837. 
Leite, V. J. S., Montagner, V. F., Ramos, D. C. W. e Peres, P. L. D. (2002). Estabilidade robusta de sistemas contínuos no tempo através de desigualdades matriciais lineares, XIV Congresso Brasileiro de Automática, Natal, RN, pp. 2878-2883.

Mori, T. e Kokame, H. (2000). A parameter-dependent Lyapunov function for a polytope of matrices, IEEE Transactions on Automatic Control 45(8): 1516-1519.

Palhares, R. M. e Peres, P. L. D. (1999). Robust $\mathcal{H}_{\infty}$ filtering design with pole placement constraint via LMIs, Journal of Optimization Theory and Applications 102(2): 239-261.

Palhares, R. M. e Peres, P. L. D. (2000). Robust $\mathcal{H}_{\infty}$ filter design with pole constraints for discrete-time systems, Journal of The Franklin Institute 337(6): 713-723.

Palhares, R. M. e Peres, P. L. D. (2001). LMI approach to the mixed $\mathcal{H}_{2} / \mathcal{H}_{\infty}$ filtering design for discrete-time uncertain systems, IEEE Transactions on Aerospace and Electronic Systems 37(1): 292-296.

Peaucelle, D., Arzelier, D., Bachelier, O. e Bernussou, J. (2000). A new robust $\mathcal{D}$-stability condition for real convex polytopic uncertainty, Systems \& Control Letters 40(1): 21-30.

Ramos, D. C. W. e Peres, P. L. D. (2001). A less conservative LMI condition for the robust stability of discretetime uncertain systems, Systems \& Control Letters 43(5): 371-378.

Ramos, D. C. W. e Peres, P. L. D. (2002). An LMI condition for the robust stability of uncertain continuous-time linear systems, IEEE Transactions on Automatic Control 47(4): 675-678.

Rantzer, A. e Johansson, M. (2000). Piecewise linear quadratic optimal control, IEEE Transactions on Automatic Control 45(4): 629-637.

Trofino, A. (1999). Parameter dependent Lyapunov functions for a class of uncertain linear systems: an LMI approach, Proceedings of the 38th IEEE Conference on Decision and Control, Vol. 1, Phoenix, AZ, pp. 23412346.

Xie, L., Shishkin, S. e Fu, M. (1997). Piecewise Lyapunov functions for robust stability of linear time-varying systems, Systems \& Control Letters 31(3): 165-171. 